IFT-2005/2

CERN-PH-TH/2005-011

DESY-05-023

December 12, 2018

\title{
Flavour violation in general supergravity
}

\author{
Piotr H. Chankowski ${ }^{1}$, Oleg Lebedev ${ }^{2}$ and Stefan Pokorski ${ }^{1,3}$ \\ ${ }^{1}$ Institute of Theoretical Physics, Warsaw University, Hoża 69, 00-681, Warsaw, Poland \\ 2 DESY, Theory Group, Notkestraße 85, 22603 Hamburg, Germany \\ 3 Theoretical Physics Division, Physics Department, CERN, CH-1211 Geneva 23, \\ Switzerland
}

\begin{abstract}
We reappraise the flavour changing neutral currents (FCNC) problem in string-derived supergravity models. We overview and classify possible sources of flavour violation and find that the problem often does not arise in classes of models which generate hierarchical Yukawa matrices. In such models, constraints from the $K$ - and $D$-meson systems leave room for substantial flavour non-universality of the soft terms. The current $B$-physics experiments only begin to probe its natural range. Correlations among different observables can allow one to read off the chirality structure of flavour violating sources. We briefly discuss the lepton sector where the problem of FCNC is indeed serious and perhaps points at an additional symmetry or flavour universality.
\end{abstract}




\section{Introduction}

The purpose of this paper is to classify different sources of flavour violation in supergravity (SUGRA) theories and study the FCNC problem associated with these sources. Concerning the latter, it is important to ask the "right" question, that is, how problematic are the FCNC in models that successfully generate the Yukawa matrices rather than in some "generic" framework.

The strategy we pursue in this paper is as follows. First, we classify various sources of flavour violation in general supergravity models. We concentrate on certain "benchmark" textures of the soft terms. Then, using a number of representative Yukawa textures, we evolve the soft terms to low energies and study implications of the generated flavour changing neutral currents. In some cases such as models with flavour violation through the Kähler potential or democratic Yukawa textures, the problem is severe. On the other hand, when flavour structures are due to different localizations of matter fields in the compact space, the FCNC are well suppressed. This is in fact a common situation in string models which produce the Yukawa coupling hierarchy (e.g. heterotic string or intersecting brane models). In string theory, the mechanisms that can generate the fermion mass hierarchy are quite constraining. As a result, the soft supersymmetry (SUSY) breaking terms in superstring derived SUGRA models are of special forms and the problem of FCNC becomes mild or simply disappears. A natural consequence of such models is that the mixing between the second and third generation squarks is expected to be substantial and to lead to effects potentially detectable at $B$-factories.

We briefly discuss the lepton sector in which a different picture emerges and the expected flavour violation exceeds the experimental limits. This perhaps signals an additional symmetry or flavour universality of the soft terms, which will be probed further in the upcoming round of $\mu \rightarrow e \gamma$ experiments.

Finally, we discuss how correlations among various $B$-physics observables would allow one to pinpoint the source of flavour violation.

\section{Classification of flavour-violating sources in super- gravity}

The $N=1$ supergravity Lagrangian is determined by 3 functions: the Kähler potential $K$, the superpotential $W$ and the gauge kinetic function $f$ (see [1] for a review). These are functions of the hidden and observable sector fields. Since the characteristic mass scale of the observable fields $\phi^{\alpha}$ is much smaller than the Planck scale, one can expand $K$ and $W$ as

$$
K=\hat{K}+\tilde{K}_{\bar{\alpha} \beta} \phi^{* \bar{\alpha}} \phi^{\beta}+\left(\frac{1}{2} Z_{\alpha \beta} \phi^{\alpha} \phi^{\beta}+\text { H.c. }\right)+\ldots
$$




$$
W=\hat{W}+\frac{1}{2} \mu_{\alpha \beta} \phi^{\alpha} \phi^{\beta}+\frac{1}{6} \mathcal{Y}_{\alpha \beta \gamma} \phi^{\alpha} \phi^{\beta} \phi^{\gamma}+\ldots
$$

where all parameters are generally functions of the hidden sector fields. Once the hidden sector fields develop (large) vacuum expectation values, these parameters will play a role of various couplings in the observable sector. Generally, the resulting kinetic terms for the observable sector fields are neither canonical nor diagonal, so in order to obtain physical fields, further diagonalization and rescaling are required.

The soft SUSY breaking terms are obtained from the general supergravity scalar potential by fixing the gravitino mass $m_{3 / 2}$ and the VEVs of the hidden sector fields, while sending the Planck mass to infinity, $M_{\mathrm{Pl}} \rightarrow \infty[2,3$. The fermion Yukawa couplings are then rescaled as

$$
\mathcal{Y}_{\alpha \beta \gamma}^{\prime}=\frac{\hat{W}^{*}}{|\hat{W}|} e^{\hat{K} / 2} \mathcal{Y}_{\alpha \beta \gamma} .
$$

The resulting soft SUSY breaking potential relevant to flavour physics is given by [4]

$$
V_{\text {soft }}=m_{\bar{\alpha} \beta}^{2} \phi^{* \bar{\alpha}} \phi^{\beta}+\left(\frac{1}{6} \mathcal{A}_{\alpha \beta \gamma} \phi^{\alpha} \phi^{\beta} \phi^{\gamma}+\text { H.c. }\right)
$$

where

$$
\begin{aligned}
m_{\bar{\alpha} \beta}^{2} & =\left(m_{3 / 2}^{2}+V_{0}\right) \tilde{K}_{\bar{\alpha} \beta}-\bar{F}^{\bar{m}} F^{n}\left(\partial_{\bar{m}} \partial_{n} \tilde{K}_{\bar{\alpha} \beta}-\tilde{K}^{\gamma \bar{\delta}} \partial_{\bar{m}} \tilde{K}_{\bar{\alpha} \gamma} \partial_{n} \tilde{K}_{\bar{\delta} \beta}\right) \\
\mathcal{A}_{\alpha \beta \gamma} & =\frac{\hat{W}^{*}}{|\hat{W}|} e^{\hat{K} / 2} F^{m}\left[\hat{K}_{m} \mathcal{Y}_{\alpha \beta \gamma}+\partial_{m} \mathcal{Y}_{\alpha \beta \gamma}\right. \\
& \left.-\left(\tilde{K}^{\delta \bar{\rho}} \partial_{m} \tilde{K}_{\bar{\rho} \alpha} \mathcal{Y}_{\delta \beta \gamma}+(\alpha \leftrightarrow \beta)+(\alpha \leftrightarrow \gamma)\right)\right] .
\end{aligned}
$$

Here $V_{0}$ is the vacuum energy, $\partial_{m}$ denotes differentiation with respect to the $m$-th hidden sector field $\left(\hat{K}_{m} \equiv \partial_{m} \hat{K}\right), F^{m}$ are the SUSY breaking $F$-terms and $\tilde{K}^{\gamma \bar{\delta}}$ is the inverse of the Kähler metric $\tilde{K}_{\bar{\delta} \gamma}$.

Let us now make our notation more transparent. Greek indices $\alpha, \ldots$ run over all MSSM $^{1}$ superfields. However, only fields with appropriate quantum numbers can couple together. For instance, the Kähler mixing is allowed only for fields with the same SM quantum numbers, i.e. only intergenerational mixings of $Q_{i}, U_{i}, D_{i}(i=1,2,3)$ are permitted. The allowed Yukawa couplings in the quark sector are of the type $Q_{i} D_{j} H_{1}$ or $Q_{i} U_{j} H_{2}$. In the Yukawa matrices and the $A$-terms, it is convenient to fix the notation as follows: the first index is to refer to the quark doublets, the second to the quark singlets, and the last to the Higgs field, e.g. $Y_{Q_{i} D_{j} H_{1}} \equiv Y_{i j}^{d}$.

Physical fields are obtained by diagonalizing and rescaling the Kähler metric responsible for kinetic terms of the observable sector fields. The canonically normalized superfields $\varphi^{a}(a=Q, D, U)$ are given by

$$
\phi^{a}=H^{a} \varphi^{a}
$$

\footnotetext{
${ }^{1}$ For a review, see $[5]$.
} 
(no summation over $a$ ), with

$$
H^{a}=V^{a} \operatorname{diag}\left(\frac{1}{\sqrt{\tilde{K}_{1}^{a}}}, \frac{1}{\sqrt{\tilde{K}_{2}^{a}}}, \frac{1}{\sqrt{\tilde{K}_{3}^{a}}}\right) .
$$

Here $V^{a}$ are $3 \times 3$ unitary matrices diagonalizing the appropriate subblocks $\tilde{K}_{\bar{\alpha} \beta}^{a}$ of the Kähler metric and $\tilde{K}_{1-3}^{a}$ are the corresponding eigenvalues,

$$
\left(V^{a}\right)^{\dagger} \tilde{K}^{a} V^{a}=\operatorname{diag}\left(\tilde{K}_{1}^{a}, \tilde{K}_{2}^{a}, \tilde{K}_{3}^{a}\right)
$$

The Yukawa couplings and the soft terms transform correspondingly, such that the seven physical $3 \times 3$ flavour structures are

$$
\begin{aligned}
Y^{u} & =\frac{1}{\sqrt{\tilde{K}_{H_{2}}}}\left(H^{q}\right)^{T} \mathcal{Y}^{u} H^{u}, \quad Y^{d}=\frac{1}{\sqrt{\tilde{K}_{H_{1}}}}\left(H^{q}\right)^{T} \mathcal{Y}^{d} H^{d}, \\
A^{u} & =\frac{1}{\sqrt{\tilde{K}_{H_{2}}}}\left(H^{q}\right)^{T} \mathcal{A}^{u} H^{u}, \quad A^{d}=\frac{1}{\sqrt{\tilde{K}_{H_{1}}}}\left(H^{q}\right)^{T} \mathcal{A}^{d} H^{d}, \\
m_{Q}^{2} & =H^{q \dagger} m_{Q}^{2} H^{q}, \quad m_{U}^{2}=H^{u \dagger} m_{U}^{2} H^{u}, \quad m_{D}^{2}=H^{d \dagger} m_{D}^{2} H^{d},
\end{aligned}
$$

where the factors $1 / \sqrt{\tilde{K}_{H_{1,2}}}$ account for rescaling of the Higgs fields. These structures are the sources of flavour violation in the MSSM. Clearly, flavour dependence in the Yukawa matrices is mandatory, whereas the soft terms serve as additional sources of flavour violation. The underlying reason for these additional sources is the Kähler potential and Yukawa couplings dependence on the hidden sector fields: indeed, as seen from Eq. (44), if the Kähler metric for observables fields and the Yukawa couplings were mere constants, the soft masses would be proportional to the unit matrix in the physical basis and the $A$-terms would be proportional to the Yukawa matrix.

Generally, flavour structures of the soft masses and the $A$-terms are independent. In particular, unlike the soft masses, the $A$-terms receive a contribution from $\partial_{m} \mathcal{Y}_{\alpha \beta \gamma}$. Thus, even if the Kähler potential is trivial, the $A$-term structure can be quite rich.

It is also manifest from Eq. (4) that a non-trivial Kähler potential generally induces flavour violation in both the soft masses and the $A$-terms. Yet, it is conceivable that such flavour violating terms may cancel out in the $A$-terms, due to some deeper dynamical reason. The soft masses would then be the only source of flavour violation (in addition to the Yukawa couplings).

In general, the soft breaking terms violate CP. CP violating phases have two sources. Firstly, they are induced by complex SUSY breaking $F$-terms, $F^{m}$, which also generate $\mathrm{CP}$ phases in flavour-independent quantities such as gaugino masses, the $\mu$-term, etc. Secondly, CP phases appear due to complex SUSY preserving quantities such as the Yukawa 
couplings and the Kähler metric ${ }^{2}$. Both sources are problematic for phenomenology, which will be discussed below.

Clearly, there are many possibilities which have different motivations and distinct phenomenology. Below we classify them.

\subsection{Flavour violation through the Yukawa couplings only}

It is possible that flavour dependence does not appear in the soft terms [7]. This occurs, for example, when

$$
F^{m} \partial_{m} \tilde{K}_{\bar{\alpha} \beta}=F^{m} \bar{F}^{\bar{n}} \partial_{m} \partial_{\bar{n}} \tilde{K}_{\bar{\alpha} \beta}=F^{m} \partial_{m} \mathcal{Y}_{\alpha \beta \gamma}=0
$$

This essentially means that the hidden sector fields that generate flavour dependence do not break supersymmetry.

The most common example of this situation is the dilaton dominated SUSY breaking scenario [3]. In this case, the only SUSY breaking field is the dilaton $S$ which produces no flavour dependence,

$$
F^{S} \neq 0, F^{m}=0 \text { for } m \neq S .
$$

Then, the soft terms are universal at the string scale and we have a version of the minimal supergravity (minimal SUGRA) model with the sfermion soft terms parametrized by $m_{0}^{2}$ and $A_{0}$ :

$$
\left(m_{Q, U, D}^{2}\right)_{i j}=m_{0}^{2} \delta_{i j}, \quad A_{i j}^{u, d}=A_{0} Y_{i j}^{u, d}
$$

\subsection{Additional flavour violation in $A$-terms only}

If, for example,

$$
F^{m} \partial_{m} \mathcal{Y}_{\alpha \beta \gamma} \not \subset \mathcal{Y}_{\alpha \beta \gamma}
$$

but

$$
F^{m} \partial_{m} \tilde{K}_{\bar{\alpha} \beta} \propto F^{m} \bar{F}^{\bar{n}} \partial_{m} \partial_{\bar{n}} \tilde{K}_{\bar{\alpha} \beta} \propto \tilde{K}_{\bar{\alpha} \beta}
$$

flavour dependence appears only in the $A$-terms [8]. This means that the hidden sector fields responsible for flavour dependence of the Kähler potential (if present at all) do not break supersymmetry and, moreover, are different from those generating flavour dependence of the Yukawa couplings.

This is a rather common situation in string models. Indeed, the $A$-terms are trilinear parameters and are closely related to the Yukawa couplings, whereas the soft masses are bilinear and more akin to the Kähler potential. Thus, generally, they are not directly related to each other.

\footnotetext{
${ }^{2}$ The reparametrization invariant measures of $\mathrm{CP}$ violation are given by quantities of the type $\operatorname{Arg}\left(\mathcal{A}_{\alpha \beta \gamma}^{*} \mathcal{Y}_{\alpha \beta \gamma}\right)[6]$. These can be non-vanishing even if all $F^{m}$ are real.
} 
For example, in the heterotic string models the Yukawa hierarchies are naturally produced if the matter fields are twisted [9]-[11], i.e. localized at special points in the compactified space. In such models, the hidden sector field that enters the Yukawa coupling is the $T$-modulus, $\mathcal{Y}_{\alpha \beta \gamma}=\mathcal{Y}_{\alpha \beta \gamma}(T)$. Generally,

$$
F^{T} \neq 0
$$

and $\partial_{m} \mathcal{Y}_{\alpha \beta \gamma}$ are not proportional to $\mathcal{Y}_{\alpha \beta \gamma}$, which leads to non-trivial $A$-terms. On the other hand, the Kähler metric is diagonal for twisted states and depends on modular weights of these states:

$$
\tilde{K}_{\bar{\alpha} \beta}=\delta_{\bar{\alpha} \beta}(T+\bar{T})^{n_{\bar{\alpha}}}
$$

The modular weights $n_{\alpha}$ are constrained by the string selection rules for the Yukawa couplings (see e.g. [12]) and are typically generation-independent. The reason is that to obtain a non-trivial structure of the Yukawa couplings and/or CP violation at the renormalizable level often requires the quark fields of different generations to belong to the same twisted sector ${ }^{3}$ (see e.g. [11, 13, 14]). Consequently, these quark fields have the same modular weights. (In any case, the modular weights can only be -1 or -2 for nonoscillator states [15]. Oscillator states usually correspond to SM singlets.) As a result, in these models the Kähler metric is generation-independent. The Yukawa couplings at $\langle T\rangle \sim 1$ are given by

$$
\mathcal{Y}_{\alpha \beta \gamma} \sim e^{-\kappa_{\alpha \beta \gamma} T}
$$

with order one coefficients $\kappa_{\alpha \beta \gamma}$, and a nontrivial flavour structure of the $A$-terms results from

$$
\Delta \mathcal{A}_{\alpha \beta \gamma} \sim \kappa_{\alpha \beta \gamma} \mathcal{Y}_{\alpha \beta \gamma} F^{T}
$$

Analogous results hold for the Yukawa matrices generated by the Froggatt-Nielsen mechanism, in which case $U(1)$ charges play the role of $\kappa_{\alpha \beta \gamma}$.

Similarly, in semirealistic brane models (for a review, see [16]) the Kähler metric for matter fields is often diagonal and generation-independent. For instance, replication of families naturally appears in intersecting brane models with different generations located at different intersections of the same branes [17]-[19]. The Kähler metric is then diagonal and depends on the intersection angles (and moduli) 20] but is the same for fields of the same type belonging to different generations. Thus, the situation here is similar to the one in the heterotic string case.

The resulting soft masses are generation-independent at the string scale (although they can generally be different for up and down squarks, and for left and right squarks), while the $A$-terms can have a rich flavour structure due to Eq. (12). Thus, the string scale soft breaking lagrangian is parametrized by

$$
\left(m_{Q, U, D}^{2}\right)_{i j}=m_{Q, U, D}^{2} \delta_{i j}, \quad A_{i j}^{u, d},
$$

in addition to flavour-independent parameters.

\footnotetext{
${ }^{3}$ This is always true in prime orbifolds since there is only one twisted sector.
} 


\subsection{Additional flavour violation in the Kähler potential only}

If the Kähler metric has a non-trivial generation dependence, e.g.

$$
F^{m} \partial_{m} \tilde{K}_{\bar{\alpha} \beta} \not \subset \tilde{K}_{\bar{\alpha} \beta},
$$

both the scalar masses and the $A$-terms have a non-trivial flavour structure. This situation can occur, for example, in the heterotic string if the quark field modular weights $n_{\alpha}$ are generation-dependent (see Eq. (15)). The non-trivial flavour structures arise then from

$$
\begin{aligned}
& \Delta m_{\bar{\alpha} \beta}^{2}=n_{\bar{\alpha}} \tilde{K}_{\bar{\alpha} \beta} \frac{\left|F^{T}\right|^{2}}{(T+\bar{T})^{2}}, \\
& \Delta \mathcal{A}_{\alpha \beta \gamma}=-\left(n_{\alpha}+n_{\beta}+n_{\gamma}\right) \mathcal{Y}_{\alpha \beta \gamma} \frac{F^{T}}{T+\bar{T}} \frac{\hat{W}^{*}}{|\hat{W}|} e^{\hat{K} / 2} .
\end{aligned}
$$

Here the modular weights are order one integers. Their typical values are -1 and -2 due to non-oscillator nature of the SM matter.

The Kähler metric is the only source of SUSY flavour violation if

$$
F^{m} \partial_{m} \mathcal{Y}_{\alpha \beta \gamma} \propto \mathcal{Y}_{\alpha \beta \gamma}
$$

For example, the contribution $F^{m} \partial_{m} \mathcal{Y}_{\alpha \beta \gamma}$ to the $A$-terms vanishes if the Yukawa structure is generated as in the Froggatt-Nielsen models [21] by non-renormalizable couplings through the vacuum expectation value of a scalar field $\phi$ which does not break supersymmetry. One then has

$$
\mathcal{Y}_{\alpha \beta \gamma}=\mathcal{Y}_{\alpha \beta \gamma}(\phi), \quad F^{\phi}=0
$$

Since in the case discussed above the Kähler metric is diagonal, the soft mass terms are also diagonal, but generically nonuniversal. In contrast, the $A$-terms can be quite complicated due to the generation dependence of the modular weights (see e.g. [22]). More complicated, non-diagonal soft mass terms can be obtained, for instance, in compactifications of the ten dimensional heterotic string on $\left(T_{2} / Z_{3}\right)^{3}$ orbifolds, where $T_{2} / Z_{3}$ are compact complex spaces ("planes") obtained by dividing complex tori $T_{2}$ by a discrete group $Z_{3}$. In this case, the three generations of untwisted matter superfields can be associated with the three "planes" $T_{2} / Z_{3}$ according to their holomorphic indices. The Kähler metric is then non-diagonal and is given by

$$
\tilde{K}_{\bar{\alpha} \beta}=\left(\operatorname{Re} M_{\bar{\alpha} \beta}\right)^{-1},
$$

where $\alpha, \beta=1,2,3$ and the 9 moduli $M_{\bar{\alpha} \beta}$ parametrize the sizes of the compactification tori and the angles between the three "planes" $T_{2} / Z_{3}$ (see e.g. [11]). In such models, the soft terms will have a richer non-diagonal flavour structure depending on specific values of the moduli. 
An interesting possibility is that non-trivial flavour dependence drops out of the $A$-terms, but remains in the soft mass terms. This occurs, for instance, if the vacuum expectation values of the moduli fields take on special values such that

$$
\begin{gathered}
F^{m} \partial_{m} \tilde{K}_{\bar{\alpha} \beta} \propto \tilde{K}_{\bar{\alpha} \beta}, \quad F^{m} \partial_{m} \mathcal{Y}_{\alpha \beta \gamma} \propto \mathcal{Y}_{\alpha \beta \gamma}, \\
F^{m} \bar{F}^{\bar{n}} \partial_{m} \partial_{\bar{n}} \tilde{K}_{\bar{\alpha} \beta} \not \tilde{K}_{\bar{\alpha} \beta}
\end{gathered}
$$

and the flavour dependence appears only through the second derivatives. Then, the $A$-terms are proportional to the Yukawa couplings while the soft masses are general.

We conclude that flavour violation through the Kähler potential generally leads to a complicated non-diagonal structure of the soft terms. The squark mass matrices and the $A$-terms are correlated, although this correlation can be far from transparent. The string scale soft breaking lagrangian is parametrized by

$$
\left(m_{Q, U, D}^{2}\right)_{i j}, \quad A_{i j}^{u, d} .
$$

Two interesting special cases are: (i) diagonal soft masses with general $A$-terms,

$$
\left(m_{Q, U, D}^{2}\right)_{i j}=\left(m_{Q, U, D}^{2}\right)_{i} \delta_{i j}, \quad A_{i j}^{u, d}
$$

as in Eq. (20), and (ii) general soft masses with universal $A$-terms,

$$
\left(m_{Q, U, D}^{2}\right)_{i j}, \quad A_{i j}^{u, d}=A^{u, d} Y_{i j}^{u, d}
$$

as in Eq. (24).

\subsection{Both additional sources present}

This is a general case and not much can be said here apart from what already appears in Eq. (41). Special cases have been covered in previous subsections. The string scale soft breaking lagrangian is general and is parametrized by

$$
\left(m_{Q, U, D}^{2}\right)_{i j}, \quad A_{i j}^{u, d} .
$$

\subsection{Summary of the textures}

The above discussion leads us to the following supergravity benchmark textures:
(A) : complete universality :
$m_{0}^{2}, A_{0}$
(B) : generation independent scalar masses and general $A-$ terms : 


$$
m_{Q}^{2}, m_{U}^{2}, m_{D}^{2}, A_{i j}^{u}, A_{i j}^{d}
$$

(C) : diagonal scalar masses and universal $A$ - terms :

$\left(m_{Q}^{2}\right)_{i},\left(m_{U}^{2}\right)_{i},\left(m_{D}^{2}\right)_{i}, A^{u}, A^{d}$

(D) : diagonal scalar masses and general $A$ - terms :

$$
\left(m_{Q}^{2}\right)_{i},\left(m_{U}^{2}\right)_{i},\left(m_{D}^{2}\right)_{i}, A_{i j}^{u}, A_{i j}^{d}
$$

(E) : general soft terms :

$$
\left(m_{Q}^{2}\right)_{i j},\left(m_{U}^{2}\right)_{i j},\left(m_{D}^{2}\right)_{i j}, A_{i j}^{u}, A_{i j}^{d} .
$$

Textures (D) and (E) also include the possibility that the soft masses and the $A$-terms are correlated, as in the case of flavour violation through the Kähler potential (Eq. (20) ). As texture (C) we choose a restricted version of the Ansatz (27) with diagonal squark masses. Here we neglect supergravity radiative corrections which can be considerable in certain models [23].

In what follows, we will study experimental constraints on the above textures and discuss how to distinguish them. They serve as boundary conditions at high energies and evolve with the energy scale. At the electroweak scale, each texture leads to a specific pattern of the mass insertions (or, more generally, flavour matrices at the interaction vertices). The main features of the resulting patterns can be summarized as follows:

(A): very little flavour changing

(B): small LL, RR and significant LR, RL flavour changing

(C): substantial LL, RR and small LR, RL flavour changing

(D): substantial LL, RR, LR, RL flavour changing

(E): substantial LL, RR, LR, RL flavour changing

Here LL and RR refer to chirality conserving flavor changing transitions in the leftand right-handed sectors, respectively. LR and RL refer to chirality flipping flavor changing transitions. For textures (C) and (D), LL/RR flavour changing results from nonuniversality of the soft masses at the string scale, that is, their departure from the form (18). Note that order one non-universality applies to $m_{i}^{2}$ rather than $m_{i}$, which makes a considerable difference for the FCNC analysis.

Since different physical processes are sensitive to different types of mass insertions, the above textures (perhaps except for (D) and (E)) are distinguishable given enough experimental information. 


\section{Low energy effects of the textures}

In this section, we recall the steps which are necessary to obtain the low energy manifestations of textures (A) to (E) and discuss their main consequences. First of all, as we have already mentioned, each texture evolves with the energy scale and this evolution is described by the renormalization group ( $R G$ ) equations. Different soft supersymmetry breaking terms evolve differently. The evolution from the GUT scale down to the electroweak scale mainly amounts to adding flavour-universal contributions to the squark mass matrices and the A-terms. These are due to gluino loops and grow with the gluino mass. The main effect of these contributions is that the average squark masse $\tilde{M}$ increases significantly. This has two important consequences: firstly, the mass insertions decrease as $1 / \tilde{M}^{2}$ and, secondly, the bounds on the mass insertions relax as $\tilde{M}$ or $\tilde{M}^{2}$. Both of these effects make the FCNC problem milder [24].

To deal with complicated flavour structures it is convenient to employ the mass insertion approximation [25] (although sometimes it may not be precise enough). The mass insertions are defined in the super-CKM basis, i.e. the basis in which the quark mass matrices are diagonal and positive,

$$
\begin{aligned}
& Y^{u} \longrightarrow V_{L}^{u \dagger} Y^{u} V_{R}^{u}=\operatorname{diag}\left(h_{u}, h_{c}, h_{t}\right), \\
& Y^{d} \longrightarrow V_{L}^{d \dagger} Y^{d} V_{R}^{d}=\operatorname{diag}\left(h_{d}, h_{s}, h_{b}\right),
\end{aligned}
$$

where $h_{i}$ denote the physical quark Yukawa couplings. To preserve the diagonal flavour structure of the supergauge vertices, the squark fields are rotated in the same fashion as the quark fields. Thus, we have the following superfield transformations:

$$
\begin{aligned}
& \hat{U}_{L, R} \longrightarrow V_{L, R}^{u} \hat{U}_{L, R}, \\
& \hat{D}_{L, R} \longrightarrow V_{L, R}^{d} \hat{D}_{L, R} .
\end{aligned}
$$

In this basis, the mass insertions at the electroweak scale are given by

$$
\left(\delta_{X Y}^{u}\right)_{i j} \equiv \frac{\left(\mathcal{M}_{X Y}^{u}\right)_{i j}^{2}}{\tilde{M}^{2}}, \quad\left(\delta_{X Y}^{d}\right)_{i j} \equiv \frac{\left(\mathcal{M}_{X Y}^{d}\right)_{i j}^{2}}{\tilde{M}^{2}}, \quad i \neq j,
$$

where $\mathcal{M}_{L L}^{u, d}, \mathcal{M}_{R R}^{u, d}, \mathcal{M}_{L R}^{u, d}$ and $\mathcal{M}_{R L}^{u, d}$ are the $3 \times 3$ blocks of the full up and down squark mass squared matrices (see e.g. [26]) and $\tilde{M}$ is the "average" squark mass appropriate for a given mass insertion. The mass insertion approximation works well when the mass insertions are significantly smaller than unity and the splittings among the eigenvalues of the mass matrix are significantly smaller than the eigenvalues themselves. The squark propagator in the mass insertion approximation has an expansion

$$
\begin{aligned}
\left\langle\tilde{q}_{\alpha} \tilde{q}_{\beta}^{*}\right\rangle & =\frac{i}{\left(k^{2} \mathbf{1}-\tilde{M}^{2} \mathbf{1}-\delta m^{2}\right)_{\alpha \beta}} \\
& =\frac{i}{k^{2}-\tilde{M}^{2}} \mathbf{1}_{\alpha \beta}+\frac{i}{\left(k^{2}-\tilde{M}^{2}\right)^{2}} \delta m_{\alpha \beta}^{2}+\frac{i}{\left(k^{2}-\tilde{M}^{2}\right)^{3}} \delta m_{\alpha \gamma}^{2} \delta m_{\gamma \beta}^{2}+\ldots,
\end{aligned}
$$


where $\alpha, \beta$ are indices of the $6 \times 6$ mass matrices. If the linear in $\delta m_{\alpha \beta}^{2}$ term happens to vanish for some $\alpha, \beta$, the leading contribution is provided by the "effective" mass insertion $\tilde{\delta}_{\alpha \beta} \sim \delta_{\alpha \gamma} \delta_{\gamma \beta}$. However, this combination is not completely equivalent to a single mass insertion $\tilde{\delta}_{\alpha \beta}$ due to a different momentum dependence of the relevant loop integral.

It is important to note that since the super-CKM basis is defined only up to a phase, one must also fix the CKM phase convention [6], which we take to be of the Wolfenstein type.

Let us now discuss some features of SUSY flavour structures in the super-CKM basis.

\subsection{A-terms in the super-CKM basis}

In order to study the LR sector, it is convenient to factor out the Yukawa couplings from the $A$-terms (see Eq. (41)),

$$
A_{i j}^{u, d} \equiv \tilde{A}_{i j}^{u, d} Y_{i j}^{u, d}
$$

Deviations from universality are then encoded in the matrix $\tilde{A}_{i j}^{u, d}$, which in the universal case has all entries equal: $\tilde{A}_{i j}^{u, d}=\tilde{A}^{u, d}$.

Unlike $A_{i j}^{u, d}$, matrix elements of $\tilde{A}_{i j}^{u, d}$ are typically $\mathcal{O}(1)$ times an overall scale factor [8], which is determined by the SUSY breaking scale and is usually of the order of the gravitino mass $m_{3 / 2}$. For instance, in the heterotic string $Y_{i j} \sim e^{-\alpha_{i j} T}$ with $\alpha_{i j}$ of order one and $T$ being the vacuum expectation value of the $T$-modulus. The non-universality of $\tilde{A}_{i j}$ is in this case given by $\partial_{T} \ln Y_{i j} \sim \alpha_{i j}$, or, for non-universal modular weights $n_{i}$, by $n_{i}+n_{j}$. In the case of the Froggatt-Nielsen mechanism, the role of $\alpha_{i j}$ 's is played by the $U(1)_{X}$ charges, with the same conclusion [27]-29]. The amount of non-universality may reduce for democratic Yukawa textures, due to smaller values of the effective $\alpha_{i j}$ 's, but this is a model-dependent issue.

The $A$-terms undergo RG evolution to low energies with the dominant contribution coming from gluino loops, which has an "aligning" effect similar to those for the squark mass matrices. Upon going over to the super-CKM basis, the $A$-terms transform just as the Yukawa matrices do,

$$
\begin{aligned}
& A_{i j}^{u} \longrightarrow\left(V_{L}^{u \dagger} A^{u} V_{R}^{u}\right)_{i j}, \\
& A_{i j}^{d} \longrightarrow\left(V_{L}^{d \dagger} A^{d} V_{R}^{d}\right)_{i j} .
\end{aligned}
$$

In this basis, $A_{i j}^{u, d}$ are generally non-diagonal and the resulting flavour-changing mass insertions are $(i \neq j)$ :

$$
\left(\delta_{L R}^{u, d}\right)_{i j}=\frac{\left.v_{u, d} A_{i j}^{u, d}\right|_{S C K M}}{\tilde{M}^{2}}
$$


where $v_{u, d}$ are the Higgs VEVs and $\tilde{M}$ is the appropriate average squark mass at the electroweak scale. To discuss orders of magnitude of various insertions it is instructive to write

$$
\left.A_{i j}^{u}\right|_{S C K M}=\text { scale factor } \times\left(\alpha_{i j} h_{u}+\beta_{i j} h_{c}+\gamma_{i j} h_{t}\right),
$$

and similarly for the down sector. Here $\alpha_{i j}, \beta_{i j}, \gamma_{i j}$ are model dependent $\leq \mathcal{O}(1)$ coefficients parametrizing departure from universality. In the universal case,

$$
\begin{aligned}
& \alpha_{11}=1 \text { and } 0 \text { otherwise } \\
& \beta_{22}=1 \text { and } 0 \text { otherwise } \\
& \gamma_{33}=1 \text { and } 0 \text { otherwise }
\end{aligned}
$$

This representation of the $A$-terms is useful for estimating typical magnitudes of the LR mass insertions. Since $\tilde{A}_{i j}^{u, d}$ are of order unity, $A_{i j}^{u, d}$ have a similar structure to that of the Yukawa matrices and the misalignment is characterized by deviation of $\alpha_{i j}, \beta_{i j}, \gamma_{i j}$ from the universal limit (37). This deviation is expected to be small if the Yukawa matrices and the $A$-terms are diagonalized by small angle rotations, which is often the case for hierarchical Yukawa textures. This ceases to be true in the case of democratic textures.

An interesting limiting case is matrix-factorizable $A$-terms, i.e. such that they can be written as

$$
A^{u, d} \equiv \tilde{B}^{u, d} \cdot Y^{u, d}+Y^{u, d} \cdot \tilde{C}^{u, d}
$$

in the matrix sense with $\tilde{B}_{i j}^{u, d}$ and $\tilde{C}_{i j}^{u, d} \lesssim \mathcal{O}(1)$ times a scale factor. In this case,

$$
\left.A^{u}\right|_{S C K M}=\left.\tilde{B}^{u}\right|_{S C K M} \cdot \operatorname{diag}\left(h_{u}, h_{c}, h_{t}\right)+\left.\operatorname{diag}\left(h_{u}, h_{c}, h_{t}\right) \cdot \tilde{C}^{u}\right|_{S C K M}
$$

and similarly for $A^{d}$. Here $\left.\tilde{B}^{u}\right|_{S C K M}$ and $\left.\tilde{C}^{u}\right|_{S C K M}$ have elements of order $\lesssim \mathcal{O}(1)$, again up to an overall scale. This implies, for instance, that the (12) element contains contributions only from $h_{u}$ and $h_{c}$, the (11) element - only from $h_{u}$, etc., and

$$
\left.A_{i j}^{u}\right|_{S C K M} \leq \text { scale factor } \times\left(\mathcal{O}(1) h_{i}+\mathcal{O}(1) h_{j}\right) .
$$

This limits the magnitude of the LR mass insertions for the first two generations and makes the SUSY FCNC problem less severe. Obviously, this situation occurs in the universal case. Other examples include models in which the Yukawa hierarchy is produced via a Froggatt-Nielsen field and models with non-universal modular weights (Eq20), such that $\tilde{A}_{i j}=a_{i}+b_{j}$ (see also [30]). We also note that the form (40) is favoured by the absence of charge and color breaking minima in the scalar potential [31].

Finally, a useful estimate of the mass insertions is obtained by setting the overall scale of the $A$-terms (and the $\mu$-term) to be equal to the average squark mass. Then, for the up sector,

$$
\left(\delta_{L R}^{u}\right)_{i j} \sim \alpha_{i j} \frac{m_{u}}{\tilde{M}}+\beta_{i j} \frac{m_{c}}{\tilde{M}}+\gamma_{i j} \frac{m_{t}}{\tilde{M}} .
$$




\subsection{LL and RR sectors in the super-CKM basis}

Upon going to the super-CKM basis, the LL and RR blocks of the squark mass matrix are rotated as

$$
\begin{aligned}
& \left(\mathcal{M}^{u, d}\right)_{L L}^{2} \longrightarrow V_{L}^{u, d \dagger}\left(\mathcal{M}^{u, d}\right)_{L L}^{2} V_{L}^{u, d}, \\
& \left(\mathcal{M}^{u, d}\right)_{R R}^{2} \longrightarrow V_{R}^{u, d \dagger}\left(\mathcal{M}^{u, d}\right)_{R R}^{2} V_{R}^{u, d} .
\end{aligned}
$$

The squark mass squared matrices $\left(\mathcal{M}^{u, d}\right)_{L L}^{2}$ and $\left(\mathcal{M}^{u, d}\right)_{R R}^{2}$ at the electroweak scale are determined by the original textures (A)-(E) and by the RG evolution. For textures (A)(D), these matrices are diagonal and therefore remain approximately diagonal after the RG evolution. Flavour violation at the electroweak scale is due to the rotations to the superCKM basis. We can easily estimate the order of magnitude of the expected effects. Let us assume for a moment that the first two and the third generations do not communicate and consider the (12) block,

$$
\mathcal{M}_{L L}^{2}=\left(\begin{array}{cc}
m_{1}^{2} & 0 \\
0 & m_{2}^{2}
\end{array}\right) .
$$

Parametrizing the orthogonal rotation matrix $V_{L}$ by $\cos \theta$ and $\sin \theta$ in the usual fashion, we get

$$
\left(\delta_{L L}\right)_{12}=\cos \theta \sin \theta \frac{\Delta m^{2}}{\tilde{M}^{2}}
$$

in the super-CKM basis, with $\Delta m^{2} \equiv m_{1}^{2}-m_{2}^{2}$ and $\tilde{M}^{2} \equiv \frac{1}{2}\left(m_{1}^{2}+m_{2}^{2}\right)$. Thus, small mass insertions are obtained for nearly degenerate masses $m_{1}$ and $m_{2}$ and/or for a small rotation angle $\theta$.

Consider now the case of 3 generations with the first two being degenerate,

$$
\mathcal{M}_{L L}^{2}=\left(\begin{array}{ccc}
m_{1}^{2} & 0 & 0 \\
0 & m_{1}^{2} & 0 \\
0 & 0 & m_{3}^{2}
\end{array}\right) .
$$

When the rotation matrix $V_{L}$ is well approximated by the CKM matrix, we have

$$
\begin{aligned}
& \left(\delta_{L L}\right)_{12} \sim 10^{-4} e^{i \mathcal{O}(1)} \frac{\Delta m^{2}}{\tilde{M}^{2}}, \\
& \left(\delta_{L L}\right)_{13} \sim 10^{-3} e^{i \mathcal{O}(1)} \frac{\Delta m^{2}}{\tilde{M}^{2}}, \\
& \left(\delta_{L L}\right)_{23} \sim 10^{-2} e^{i \mathcal{O}\left(10^{-1}\right)} \frac{\Delta m^{2}}{\tilde{M}^{2}},
\end{aligned}
$$

where $\Delta m^{2} \equiv m_{1}^{2}-m_{3}^{2}$ and $\tilde{M}$ is the average squark mass. This gives a good idea of the expected magnitude of mass insertions in the case of a small angle rotation, but can be drastically different for textures requiring a large angle rotation.

As we will see, FCNC constraints require mass insertions in the LL and RR sectors to be quite small but, nevertheless, leave room for departures from degeneracy of the eigenvalues, particularly for small rotation angles. 
In the most general texture (E), the flavour off-diagonal entries of the squark mass squared matrices are present already at the high energy scale. Their RG evolution is not important, so at the electroweak scale they remain of similar order of magnitude. Furthermore, barring accidental cancellations, the rotations to the super-CKM basis do not change the qualitative picture. The only important effect is the increase of the flavourdiagonal entries due to the RG running, which reduces the magnitude of the mass insertions. Taking this into account, experimental constraints on the off-diagonal LL and $\mathrm{RR}$ mass insertions can be applied directly to the high-energy texture (E). The result is that such insertions have to be small and generic textures (E) are inconsistent with experiment.

It is possible that the soft breaking terms and the Yukawa matrices align due to some horizontal symmetry, resulting in suppressed mass insertions. In this paper, we will take a conservative view and will not pursue this option further.

\subsection{Yukawa textures}

An important issue to address is dependence of SUSY FCNC on Yukawa textures. To cover both ends of the spectrum, we take a few representative examples with both small and large angle rotations.

\section{(i). The simplest texture.}

The simplest texture contains no extra parameters beyond those already present in the CKM matrix and quark masses: ${ }^{4}$

$$
\begin{aligned}
Y^{u} & =\frac{1}{v \sin \beta} \operatorname{diag}\left(m_{u}, m_{c}, m_{t}\right), \\
Y^{d} & =\frac{1}{v \cos \beta} V_{C K M}^{\dagger} \operatorname{diag}\left(m_{d}, m_{s}, m_{b}\right),
\end{aligned}
$$

with $v^{2}=v_{u}^{2}+v_{d}^{2}$ and $\tan \beta=v_{u} / v_{d}$. To diagonalize these matrices, only a left handed rotation $V_{C K M}$ in the down sector is required.

\section{(ii). A hierarchical texture.}

Hierarchical textures encode the hierarchy of the quark masses in a transparent manner and arise in various models. For example, they appear in string models due to the exponential dependence of the twisted sector couplings on moduli (for a recent analysis, see [32]) or due to a Froggatt-Nielsen type mechanism [33].

For definiteness, we use a set of textures from Ref. [34]. This is an $S U(5) \times U(1)$ model with order $\mathcal{O}(1)$ coefficients chosen so that a good fit to the fermion masses and

\footnotetext{
${ }^{4}$ An alternative texture of this sort, $Y^{u} \propto V_{C K M}^{\dagger} \operatorname{diag}\left(m_{u}, m_{c}, m_{t}\right), Y^{d} \propto \operatorname{diag}\left(m_{d}, m_{s}, m_{b}\right)$ would lead to smaller FCNC effects in the down type quark sector, which is constrained by experiment stronger than the up sector.
} 
mixings is assured. The $U(1)_{X}$ charges are $q=\bar{u}=\bar{e}=(3,2,0)$ and $\bar{d}=l=(4,2,2)$. The up-quark Yukawa has a structure

$$
Y^{u}=\left(\begin{array}{ccc}
\epsilon^{6} & \epsilon^{5} & \epsilon^{3} \\
\epsilon^{5} & \epsilon^{4} & \epsilon^{2} \\
\epsilon^{3} & \epsilon^{2} & \epsilon^{0}
\end{array}\right) .
$$

The down quark Yukawa matrix $Y^{d}$ is given by

$$
Y_{i j}^{d}=\left(C_{i j}^{(1)}+\kappa C_{i j}^{(2)}\right) \epsilon^{q_{i}+\bar{d}_{j}}
$$

where $\kappa=0.3, \epsilon=0.22$ and matrices $C^{(1,2)}$ have a structure of the form

$$
C_{1,2}=\left(\begin{array}{ccc}
\epsilon^{7} & \epsilon^{5} & \epsilon^{5} \\
\epsilon^{6} & \epsilon^{4} & \epsilon^{4} \\
\epsilon^{4} & \epsilon^{2} & \epsilon^{2}
\end{array}\right)
$$

Diagonalization of the Yukawa matrices in this model requires small angle rotations in the left-handed sector and large angle rotations in the right-handed sector. The latter are correlated with the large neutrino mixing angles in GUT models [35].

\section{(iii). A democratic texture.}

A strictly democratic texture predicts one massive and two massless quarks in the up and down sectors. Realistic quark masses and mixings can be produced by a perturbation around this texture [36, 37. Democratic textures can also arise in string models, e.g. when the exponential suppression of the twisted sector couplings is not significant (see e.g. Abel et al. in [19]).

In our numerical analysis, we use democratic textures similar to those of Ref. [38,

$$
\begin{gathered}
Y^{u}=\frac{m_{t}}{3 v \sin \beta}\left(\begin{array}{ccc}
1.013 & 0.987 & 0.999 \\
0.987 & 1.013 & 0.999 \\
0.999 & 0.999 & 0.998
\end{array}\right), \\
Y^{d}=\frac{m_{b}}{3 v \cos \beta} K^{\dagger} \cdot\left(\begin{array}{ccc}
0.987 & 0.905 & 0.968 \\
0.903 & 1.212 & 1.008 \\
0.967 & 1.008 & 1
\end{array}\right) \cdot K, \\
K=\operatorname{diag}\left(1, \mathrm{e}^{-0.01 \mathrm{i}}, \mathrm{e}^{0.01 \mathrm{i}}\right) .
\end{gathered}
$$

These matrices are diagonalized by a large angle rotation. 


\section{Experimental constraints}

Before we present our numerical analysis, in this section we collect experimental constraints on various mass insertions and also discuss qualitatively their implications for textures (A)-(E).

\section{Electric dipole moments}

The electric dipole moments of the neutron and mercury atom (and of the electron, in the lepton sector) are especially sensitive to flavour conserving LR mass insertions for the first generation. In particular, the current bounds

$$
\begin{aligned}
& \left|d_{n}\right|<6 \times 10^{-26} e \mathrm{~cm}, \\
& \left|d_{\mathrm{Hg}}\right|<2 \times 10^{-28} e \mathrm{~cm}
\end{aligned}
$$

impose the constraints [39]-[41]:

$$
\left|\operatorname{Im}\left(\delta_{11}^{\{u, d\}}\right)_{L R}\right| \leq 10^{-7} \div 10^{-6} .
$$

This implies that the corresponding CP phases of the $\mu$ - and $A$-terms have to be small. Clearly, this SUSY CP problem [42] arises for all supergravity textures, including the flavour universal one. The unwanted CP phases can be suppressed in some special cases, e.g. in the dilaton dominated SUSY breaking scenario with an axionic symmetry [43] or with the help of the CP phase alignment [44], but this requires additional assumptions.

In the non-universal case, the problem becomes more severe due to additional CP phases which appear in the process of diagonalizing the quark mass matrices and the enhanced magnitude of the mass insertions [14, 8]. For example,

$$
\left(\delta_{L R}^{u}\right)_{11} \sim \frac{\tilde{A}\left(m_{u}+\varepsilon m_{c}+\varepsilon^{\prime} m_{t}\right)}{\tilde{M}^{2}},
$$

where $\tilde{A} \sim \tilde{M}$ is the "overall" scale of the $A$-terms and $\varepsilon, \varepsilon^{\prime}$ are model-dependent coefficients. This makes it more difficult to satisfy the bound (53). In this sense, the EDMs prefer some sort of universality, at least in the $A$-terms, although they are problematic in any case.

The problem could be partly solved by decoupling the first two sfermion generations (although the third generation still contributes to the EDMs through the Weinberg operator). However, it appears difficult to realize this possibility in specific models. Since the overall scale of the soft terms in SUGRA is given by $m_{3 / 2}$, a very large splitting between the masses of the first two and the third sfermion generations would require some sort of a singularity in the derivatives of the Kähler potential. In practice, this does not happen and the non-universality is governed, for instance, by order one modular weights (Eq. (15)). Another option would be to assume a large $m_{3 / 2}$ and obtain light, as required 
by naturallness, third generation sfermions via RG evolution to infrared fixed points. However, in this case it would be problematic to get light enough gauginos.

Given these difficulties, we will not attempt to resolve the SUSY CP problem and, in what follows, will simply treat the EDMs as a constraint on all of the SUGRA textures.

\section{Kaon observables}

The most important observables in the Kaon system are the Kaon mass splitting, $\Delta M_{K}$, and the parameters $\epsilon$ and $\epsilon^{\prime}$ measuring $\mathrm{CP}$ violation in Kaon decays,

$$
\begin{aligned}
& \Delta M_{K}=M_{K_{L}}-M_{K_{S}} \simeq 3.5 \times 10^{-15} \mathrm{GeV}, \\
& \epsilon=\frac{A\left(K_{L} \rightarrow \pi \pi\right)}{A\left(K_{S} \rightarrow \pi \pi\right)} \simeq 2.3 \times 10^{-3}, \\
& \epsilon^{\prime} / \epsilon=-\frac{\omega}{\sqrt{2}|\epsilon| \operatorname{Re} A_{0}}\left(\operatorname{Im} A_{0}-\frac{1}{\omega} \operatorname{Im} A_{2}\right) \simeq 1.9 \times 10^{-3},
\end{aligned}
$$

where $A_{0,2}$ are the $K \rightarrow \pi \pi$ amplitudes for the $\Delta I=1 / 2,3 / 2$ transitions and $\omega \equiv$ $\operatorname{Re} A_{2} / \operatorname{Re} A_{0} \simeq 1 / 22$.

These observables place severe constraints on new physics flavour structures. In particular, SUSY contributions mediated by gluinos and squarks generally lead to $\left|\Delta M_{K}\right|,|\epsilon|$ and $\left|\epsilon^{\prime} / \epsilon\right|$ orders of magnitude too large. For gluino and squark masses of $500 \mathrm{GeV}$, and assuming no accidental cancellations between contributions of different mass insertions, the measured $\Delta M_{K}$ imposes the following bounds on the mass insertions [39],

$$
\begin{aligned}
& \sqrt{\left|\operatorname{Re}\left(\delta_{12}^{d}\right)_{L L}^{2}\right|}<4 \times 10^{-2}, \quad \sqrt{\left|\operatorname{Re}\left(\delta_{12}^{d}\right)_{L L}\left(\delta_{12}^{d}\right)_{R R}\right|}<3 \times 10^{-3}, \\
& \sqrt{\left|\operatorname{Re}\left(\delta_{12}^{d}\right)_{L R}^{2}\right|}<4 \times 10^{-3},
\end{aligned}
$$

while the measured value of the $\epsilon$ parameter [39] imposes the bounds

$$
\begin{aligned}
& \sqrt{\left|\operatorname{Im}\left(\delta_{12}^{d}\right)_{L L}^{2}\right|}<3 \times 10^{-3}, \quad \sqrt{\left|\operatorname{Im}\left(\delta_{12}^{d}\right)_{L L}\left(\delta_{12}^{d}\right)_{R R}\right|}<2 \times 10^{-4}, \\
& \sqrt{\left|\operatorname{Im}\left(\delta_{12}^{d}\right)_{L R}^{2}\right|}<4 \times 10^{-4} .
\end{aligned}
$$

The bounds on the RR mass insertion are the same as the ones on the LL insertion. All these bounds scale as $\tilde{M}$ when the squark masses are changed. Furthermore, the charginosquark loop contributions to $\Delta M_{K}$ and $\epsilon$ impose analogous (although somewhat weaker) constraints on the up squark sector mass insertions [45]. For $\mu=M_{2}=350 \mathrm{GeV}$ and the squark masses of $500 \mathrm{GeV}$, they read

$$
\sqrt{\left|\operatorname{Re}\left(\delta_{12}^{u}\right)_{L L}^{2}\right|}<1 \times 10^{-1}, \quad \sqrt{\left|\operatorname{Im}\left(\delta_{12}^{u}\right)_{L L}^{2}\right|}<1 \times 10^{-2} .
$$


Similar constraints involving RR and LR mass insertions are much weaker because of the factors $m_{q} / M_{Z}$ suppressing the couplings of the right-chiral squarks to charginos and quarks. Finally, for the same gluino and down type squark masses as above, the measured value of the $\epsilon^{\prime}$ parameter sets the rough bounds 39

$$
\left|\operatorname{Im}\left(\delta_{12}^{d}\right)_{L L}\right|<5 \times 10^{-1}, \quad\left|\operatorname{Im}\left(\delta_{12}^{d}\right)_{L R}\right|<2 \times 10^{-5} .
$$

In addition, from the chargino-up type squark contributions to $\epsilon^{\prime}$ one obtains a rather weak limit $\left|\operatorname{Im}\left(\delta_{12}^{u}\right)_{L L}\right|<0.3$ [45] and essentially no bound on other mass insertions.

Let us now discuss implications of these constraints. Clearly, at the electroweak scale only little mixing between squarks of the first two generations is allowed. The strongest bounds on the chirality conserving mass insertions come from $\Delta M_{K}$ and $\epsilon$, while those on the chirality changing mass insertions are due to $\epsilon^{\prime}$.

In the LL and RR sectors, the allowed mass insertions are of order $10^{-2}$ or smaller. This means that the soft masses in the original basis are almost diagonal (barring alignment) and the diagonal entries are almost degenerate at low energies. In the down sector, the most conservative bounds imply that this degeneracy is at a percent level for democratic Yukawa textures and at about 10\% level for hierarchical textures. In the up sector, the mass splittings can be larger ${ }^{5}$. This implies that the LL and RR sectors are to some extent universal at low energies. As we will see in the next section, the RG running from the GUT scale to the electroweak scale has an important "aligning" effect. As a result, constraints on the high energy values of the soft parameters are milder.

The situation is very different in the LR sector. Consider the mixing of the first two generation squarks and assume that their mixing with the third generation squarks is small, $\leq 10^{-2}$. A natural magnitude of the mass insertion is then (see Eq. (41))

$$
\left(\delta_{12}^{d}\right)_{L R} \sim \alpha_{12} \frac{m_{d}}{\tilde{M}}+\beta_{12} \frac{m_{s}}{\tilde{M}} \lesssim \mathcal{O}\left(10^{-4}\right),
$$

where $\alpha_{12}, \beta_{12}$ are $\lesssim \mathcal{O}(1)$ model dependent coefficients. It follows that all the bounds except for that from $\epsilon^{\prime}$ are satisfied automatically. $\epsilon^{\prime}$ imposes a rather mild constraint on the imaginary part of $A_{12}^{d}$ (see, e.g. [46]). The same considerations also apply to the up squark sector. This means that order one non-universality is allowed in the (12) block of $\tilde{A}_{i j}^{d}$.

The above estimate holds in a wide class of models including those with hierarchical textures, matrix-factorizable A-terms, etc. However, it may not apply to the case of democratic textures, which we study below numerically.

Finally, we note that there also exist bounds on products of the mass insertions when one goes beyond a single mass insertion approximation. To give an example, charginosquark penguin diagrams with two mass insertions on the squark line modify, in particular,

\footnotetext{
${ }^{5}$ If the CKM matrix is entirely due to the up sector and the Yukawa textures are hierarchical, the constraints are particularly weak and no significant degeneracy is required. This, however, appears to be a rather special case.
} 
the effective $Z^{0} \bar{d} s$ vertex. The resulting SUSY contribution to $\mathrm{BR}\left(K^{+} \rightarrow \pi^{+} \nu \bar{\nu}\right)$ does not exceed its measured value provided $\left|\operatorname{Re}\left(\delta_{L R}^{u}\right)_{32}^{*}\left(\delta_{L R}^{u}\right)_{31}\right|<0.2$ [47]. In SUGRA models, this product is expected to be bounded by $\left(m_{t} / \tilde{M}\right)^{2} \sim 10^{-1}$ and the constraint is satisfied automatically. However, in other scenarios, large SUSY effects in the $Z^{0} \bar{d} s$ vertex are possible. They can lead, in particular, to the branching ratio of the $K_{L} \rightarrow \pi^{0} \nu \bar{\nu}$ decay by up to two orders of magnitude larger than the SM prediction [47.

\section{$D^{0}-\bar{D}^{0}$ mixing}

The experimental bound on the $D^{0}-\bar{D}^{0}$ mixing is

$$
\Delta M_{D}<4.8 \times 10^{-14} \mathrm{GeV} .
$$

For the gluino and squark masses of $500 \mathrm{GeV}$, the constraints on the up type squark mass insertions read [48]:

$$
\begin{aligned}
& \sqrt{\left|\operatorname{Re}\left(\delta_{12}^{u}\right)_{L L}^{2}\right|}<5 \times 10^{-2}, \quad \sqrt{\left|\operatorname{Re}\left(\delta_{12}^{u}\right)_{L L}\left(\delta_{12}^{u}\right)_{R R}\right|}<1 \times 10^{-2}, \\
& \sqrt{\left|\operatorname{Re}\left(\delta_{12}^{u}\right)_{L R}^{2}\right|}<2 \times 10^{-2},
\end{aligned}
$$

and they scale as $\tilde{M}$ when the squark masses are changed. The above bounds result from the gluino-up type squark contribution to $\Delta M_{D}$ and are comparable to the ones stemming from the chargino-squark contributions to the Kaon observables discussed in the previous section. Concerning the LR mass insertions in the up sector, an estimate analogous to Eq. (60) holds and we reach the same conclusion that order one non-universality in the (12) block of the $A$-terms is allowed.

\section{$B$ meson observables}

The most important constraints on the flavour changing transitions involving the $b$ quark are

$$
\begin{aligned}
& \Delta M_{B_{d}} \simeq 3.4 \times 10^{-13} \mathrm{GeV}, \\
& \operatorname{BR}\left(\bar{B} \rightarrow X_{s} \gamma\right) \simeq 3.3 \times 10^{-4}, \\
& S_{B_{d}^{0} \rightarrow \psi K_{S}} \simeq 0.73,
\end{aligned}
$$

where $S_{B_{d}^{0} \rightarrow \psi K_{S}}$ measures the CP violating asymmetry in the $B_{d}^{0} \rightarrow \psi K_{S}$ decay, proportional to $\Gamma\left(\bar{B}_{d}^{0} \rightarrow \psi K_{S}\right)-\Gamma\left(B_{d}^{0} \rightarrow \psi K_{S}\right)$.

For the gluino and squark masses of $500 \mathrm{GeV}$, gluino-squark loop contributions to $\Delta M_{B_{d}}$ lead to the following constraints on the down type squark mass insertions [39]:

$$
\begin{aligned}
& \sqrt{\left|\operatorname{Re}\left(\delta_{13}^{d}\right)_{L L}^{2}\right|}<1 \times 10^{-1}, \quad \sqrt{\left|\operatorname{Re}\left(\delta_{13}^{d}\right)_{L L}\left(\delta_{13}^{d}\right)_{R R}\right|}<2 \times 10^{-2}, \\
& \sqrt{\left|\operatorname{Re}\left(\delta_{13}^{d}\right)_{L R}^{2}\right|}<3 \times 10^{-2} .
\end{aligned}
$$


These limits scale as $\tilde{M}$ when the squark masses are changed. $S_{B_{d}^{0} \rightarrow \psi K_{S}}$ imposes similar constraints on the imaginary parts of the same combinations of the mass insertions [49]. As the value of $\Delta M_{B_{s}}$ is still not bounded from above by experiment, there are no similar limits on the 23 mass insertions. The experimental value of $\operatorname{BR}\left(\bar{B} \rightarrow X_{s} \gamma\right)$ sets a limit on the absolute value of the $\left(\delta_{23}^{d}\right)_{L R}$ mass insertion [39]:

$$
\left|\left(\delta_{23}^{d}\right)_{L R}\right|<1.5 \times 10^{-2}
$$

for the gluino and squark masses of $500 \mathrm{GeV}$ and the bound scales as $\tilde{M}^{2}$ when the squark masses are changed.

Constraints on the up sector mass insertions are quite weak: the chargino-squark contribution to $\Delta M_{B_{d}}$ leads to the bound on $\left(\delta_{13}^{u}\right)_{L L}$ [49]: $\left|\left(\delta_{13}^{u}\right)_{L L}\right| \leq \mathcal{O}\left(10^{-1}\right)$. The insertions $\left(\delta_{13}^{u}\right)_{R R},\left(\delta_{13}^{u}\right)_{L R},\left(\delta_{13}^{u}\right)_{R L}$ as well as all $\left(\delta_{23}^{u}\right)_{X Y}$ are essentially unconstrained.

In SUGRA models, the LR mass insertions connecting the third generation with the other two are generically of the order

$$
\begin{aligned}
\left(\delta_{i 3}^{d}\right)_{L R} & \lesssim \mathcal{O}\left(\frac{m_{b}}{\tilde{M}}\right) \sim 10^{-2}, \\
\left(\delta_{i 3}^{u}\right)_{L R} & \lesssim \mathcal{O}\left(\frac{m_{t}}{\tilde{M}}\right) \sim 0.1 \div 1
\end{aligned}
$$

for $i=1,2$ and the experimental constraints are satisfied automatically. Thus, the current bounds allow for order one non-universality in the $A$-terms involving the third generation. Some degree of universality is required in the chirality conserving sectors, although the constraints are much weaker than those on the mixing of the first two generations.

To conclude this section, the above simple estimates show that order one nonuniversality in the $A$-terms is consistent with the current data, whereas the soft mass terms are required to be essentially diagonal and somewhat degenerate at low energies. As explained earlier, the corresponding constraints on the high energy parameters are significantly weaker due to the aligning effect of the RG evolution. Below we confirm these conclusions numerically.

\section{$5 \quad$ Numerical results}

In this section, we present results of our numerical analysis for textures (A), (B) and (C) and compare them with the experimental constraints listed in section 4. Barring accidental cancellations, these results also apply to texture (D) which is a combination of textures (B) and (C). For texture (E), no numerical analysis is needed to see that it is inconsistent with the FCNC constraints unless it reduces to one of the special cases (A)-(D).

The numerical analysis of this section is necessary to support our qualitative conclusions of section 4. Moreover, it illustrates the dependence of the FCNC constraints on the 
chosen Yukawa textures and on squark and gluino masses. It is also important to study at a quantitative level the still remaining room for flavour dependence in the Kähler potential and the $A$-terms in supergravity models, so that prospects for further experimental investigations can be assessed.

In our analysis, we use the Yukawa textures described in section 3 and the standard 1-loop RG equations for the evolution of the soft terms from the high energy scale down to the electroweak scale. Our results are presented as a function of the high (string) scale values of the parameters and compared with the limits on mass insertions. The limits shown in the plots are properly rescaled to account for the actual values of low energy mass parameters obtained from the RG evolution.

\section{Texture (A)}

Flavour violating effects are very small, especially in the RR sector. For completeness, in Table 1 we provide representative mass insertions generated by the $\mathrm{RG}$ running.

\begin{tabular}{|c|c|c|c|}
\hline mass insertion & 12 & 13 & 23 \\
\hline $\mathrm{d}, \mathrm{LL}$ & $10^{-4}$ & $10^{-3}$ & $10^{-2}$ \\
$\mathrm{~d}, \mathrm{RR}$ & $10^{-13}$ & $10^{-10}$ & $10^{-8}$ \\
$\mathrm{~d}, \mathrm{LR}$ & $10^{-8}$ & $10^{-5}$ & $10^{-5}$ \\
$\mathrm{u}, \mathrm{LL}$ & $10^{-7}$ & $10^{-5}$ & $10^{-4}$ \\
$\mathrm{u}, \mathrm{RR}$ & $10^{-16}$ & $10^{-12}$ & $10^{-9}$ \\
$\mathrm{u}, \mathrm{LR}$ & $10^{-11}$ & $10^{-8}$ & $10^{-6}$ \\
\hline
\end{tabular}

Table 1: RG generated mass insertions for $\tan \beta=3, m_{0}=200 \mathrm{GeV}, A_{0}=50 \mathrm{GeV}$ and $M_{1 / 2}=100 \mathrm{GeV}$.

\section{Texture (B)}

The most important effects are in the LR/RL sector. Figures (11)-(3) display the relevant mass insertions and the experimental bounds for Yukawa textures (i)-(iii), respectively. At the GUT scale the gaugino masses are fixed to $200 \mathrm{GeV}$ for textures (i), (ii), and to $500 \mathrm{GeV}$ for texture (iii). The horizontal axis corresponds to the sfermion masses at the GUT scale: $m_{Q}^{2}=m_{U}^{2}=m_{D}^{2} \equiv m^{2}$. The mass scale of the $A$-terms is taken as $\tilde{A}=m / 2$ and order one non-universal entries of $\tilde{A}_{i j}$ are generated randomly. Each panel shows combinations of both the LR and the RL mass insertions, e.g. $\left|\operatorname{Re}\left(\left(\delta_{L R}^{u}\right)_{12}\left(\delta_{L R}^{u}\right)_{12}\right)\right|^{1 / 2}$ and $\left|\operatorname{Re}\left(\left(\delta_{R L}^{u}\right)_{12}\left(\delta_{R L}^{u}\right)_{12}\right)\right|^{1 / 2}$. This explains the presence of two distinct bands in some of the panels.

We see that for the hierarchical Yukawa textures (i) and (ii), the only problematic observables are the EDMs, representing the SUSY CP problem. The constraint stemming from $\epsilon^{\prime}$ is rather mild. The situation is much worse for the democratic texture (iii), for which all the limits imposed by the Kaon observables are exceeded. 
Texture $(C)$

Significant LL and RR mass insertions are induced. In Figures (4)-(9), relevant mass insertions are shown for the GUT scale boundary condition

$$
m_{Q}^{2}=m_{U}^{2}=m_{D}^{2}=\left(\begin{array}{ccc}
m_{1}^{2} & 0 & 0 \\
0 & m^{2} & 0 \\
0 & 0 & m^{2}
\end{array}\right)
$$

and Yukawa textures (i)-(iii). As before, the gaugino masses are fixed to $200 \mathrm{GeV}$ for textures (i), (ii), and to $500 \mathrm{GeV}$ for texture (iii). $m_{1}$ is generated randomly in the range $\frac{1}{2} m \div m$. (In fact, we allow for larger departures from universality than usually exists in typical semirealistic models, see Section 2).

Figures (4) -(9) show that the mass insertions grow with $m$, which is perhaps counterintuitive. The reason for that is the gluino loop renormalization effect which is more important for small values of $m$ [24]. The low-energy degeneracy parameter $\left(m^{2}-m_{1}^{2}\right) /\left(m^{2}+\right.$ $\Delta_{\tilde{g}} m^{2}$ ), where $\Delta_{\tilde{g}} m^{2}$ is induced by the RG running, grows with $m^{2}$ leading to larger mass insertions. This "aligning" gluino effect is very important and can reduce a mass insertion by up to an order of magnitude for similar squark and gluino masses. For larger gluino masses it is even more important.

For $m \sim M_{1 / 2}$ and small rotation angles (texture (i)), we see that no significant FCNC problem exists. Even if the mass splitting at the GUT scale is of order one, $\delta_{L L, R R}^{12}$ are suppressed by both the gluino loop RG effect and the small rotation angle (see Eq. (44)).

For large rotation angles in the right-handed sector (texture (ii)), the problem of FCNC becomes more acute, mainly due to the simultaneous presence of large LL and $\mathrm{RR}$ mass insertions. The most constraining observable here is $\epsilon$. The problem disappears eventually as $M_{1 / 2}$ increases, yet it still persists for $M_{1 / 2}=500 \mathrm{GeV}$. This is also true for texture (iii) as seen in Figs. (89).

An interesting "focusing" effect is seen in Figures (5) and (77). The values of $\left(\delta_{23}^{d}\right)_{L L}$ are independent of $m_{1}$ and of the specific Yukawa texture as long as it is diagonalized by a small angle rotation in the left-handed sector. This is because the dominant contribution to $\left(\delta_{23}^{d}\right)_{L L}$ is due to the top Yukawa RG effect which depends on the CKM matrix only.

Figures (10)-(15) show our results for the case of degenerate first two generations,

$$
m_{Q}^{2}=m_{U}^{2}=m_{D}^{2}=\left(\begin{array}{ccc}
m^{2} & 0 & 0 \\
0 & m^{2} & 0 \\
0 & 0 & m_{3}^{2}
\end{array}\right),
$$

with $m_{3}$ generated randomly in the range $\frac{1}{2} m \div m$. Again, no problem with FCNC arises for texture (i). For texture (ii), there is some tension with $\epsilon$ due to large rotations in the right-handed sector. The problem becomes milder with increasing gluino mass and dissappears for $M_{1 / 2}=500 \mathrm{GeV}$. The FCNC problem is serious for the Yukawa texture (iii), in which case the limits stemming from the Kaon and $D$-meson observables 
are exceeded for $M_{1 / 2}=200 \mathrm{GeV}$ (the $B_{d^{-}}^{0} \bar{B}_{d}^{0}$ mixing imposes only a mild constraint). However, no significant FCNC problem exists for heavier gaugino masses, $M_{1 / 2}=500$ $\mathrm{GeV}$, as shown in Figures (14) and (15).

For texture (D), similar conclusions can be drawn by combining the results for textures (B) and (C). The main point is that the $A$-term nonuniversality is essentially unconstrained (ignoring the $\mathrm{CP}$ problem), while some degeneracy of the diagonal soft masses may be required.

\begin{tabular}{|c||c|c|}
\hline Observable & Kähler potential flavour violation & $A$-term flavour violation \\
\hline$\Delta M_{K}$ & problem & no problem \\
$\epsilon$ & problem & no problem \\
$\epsilon^{\prime}$ & no problem & no problem \\
$\mathrm{BR}(K \rightarrow \pi \nu \bar{\nu})$ & no problem & no problem \\
$\Delta M_{D}$ & problem & no problem \\
$\Delta M_{B_{d}}$ & problem & no problem \\
$\mathrm{BR}(b \rightarrow s \gamma)$ & no problem & no problem \\
$A_{\mathrm{CP}}\left(B \rightarrow \psi K_{s}\right)$ & problem & \\
\hline
\end{tabular}

Table 2: Observables and their sensitivity to the source of flavour violation. Here "Kähler potential flavour violation" refers to misalignment between the Kähler potential and the soft scalar masses of Eq. (4) (texture (E)), while " $A$-term flavour violation" refers to misalignment between the $A$-terms and the Yukawa matrices. The entries indicate whether order one non-universality at the high energy scale is in conflict with the particular observable when hierarchical Yukawa matrices are assumed.

Finally, we compare in Table 2 SUSY flavour violation resulting from misalignment between the Kähler potential and the soft scalar masses (texture (E)) with flavour violation resulting from misalignment between the $A$-terms and the Yukawa matrices. Clearly, the former scenario is strongly constrained. This implies that the Kähler potential and the soft scalar mass terms are diagonal (to a good approximation) in the same basis. Generally, there are further constraints on the diagonal entries of the soft mass squared matrices. These constraints strongly depend on the Yukawa textures. If the Yukawa matrices are diagonalized by large angle rotations, the diagonal entries must be degenerate to a large extent. On the other hand, if the Yukawa matrices are diagonalized by rotation matrices similar to the CKM one, order one splittings among the diagonal entries are allowed.

\section{Comments on the lepton sector}

So far we have been focusing on the squark sector. In the lepton sector, the analysis becomes more involved due to unknown origin and nature of neutrino masses. For example, if they originate from the seesaw mechanism operating at some high scale $M_{R}<M_{\text {string }}$, 
one should also take into account effects of the additional Yukawa couplings which generate off-diagonal entries in the slepton mass matrices during the $\mathrm{RG}$ evolution. We do not undertake such an analysis here. Instead, we only make some qualitative remarks (neglecting the RG effects).

The most restrictive observables are the $l_{i} \rightarrow l_{j} \gamma$ branching ratios [50]:

$$
\begin{aligned}
& \operatorname{BR}(\mu \rightarrow e \gamma)<1.2 \times 10^{-11} \\
& \operatorname{BR}(\tau \rightarrow e \gamma)<2.7 \times 10^{-6} \\
& \operatorname{BR}(\tau \rightarrow \mu \gamma)<5.0 \times 10^{-7}
\end{aligned}
$$

The corresponding constraints on the mass insertions can be obtained by rescaling the original results of Ref. 39,

$$
\begin{array}{ll}
\left|\left(\delta_{12}^{l}\right)_{L L}\right|<4 \times 10^{-3}, & \left|\left(\delta_{12}^{l}\right)_{L R}\right|<1 \times 10^{-6}, \\
\left|\left(\delta_{13}^{l}\right)_{L R}\right|<2 \times 10^{-2}, & \left|\left(\delta_{23}^{l}\right)_{L R}\right|<1 \times 10^{-2}
\end{array}
$$

for the photino and slepton masses of $100 \mathrm{GeV}$. The constraint on $\left(\delta_{12}^{l}\right)_{R R}$ is the same as that on $\left(\delta_{12}^{l}\right)_{L L}$, while the other insertions are essentially unconstrained.

The bounds (70) immediately tell us that the LL and RR blocks of the slepton mass matrix in the (12) sector are proportional to the unit matrix with good accuracy. As to the LR sector, a simple estimate of $\left(\delta_{12}^{l}\right)_{L R}$ gives

$$
\left(\delta_{12}^{l}\right)_{L R} \sim \alpha_{12} \frac{m_{e}}{\tilde{M}}+\beta_{12} \frac{m_{\mu}}{\tilde{M}}+\gamma_{12} \frac{m_{\tau}}{\tilde{M}}
$$

with $\alpha_{12}, \beta_{12}, \gamma_{12} \lesssim \mathcal{O}(1)$. The bounds then imply

$$
\alpha_{12} \lesssim 10^{-1}, \quad \beta_{12} \lesssim 10^{-3}, \quad \gamma_{12} \lesssim 10^{-4}
$$

Thus, the alignment between the $A$-terms and the lepton Yukawa matrices has to be very precise, ${ }^{6}$ in sharp contrast with the squark sector. This suggests that the charged lepton Yukawa matrix is diagonal at the high energy scale, presumably due to some symmetry such as the lepton number, or that the $A$-terms are universal.

The bound on $\mathrm{BR}(\mu \rightarrow e \gamma)$ is expected to be improved by 3 orders of magnitude at PSI. The above estimates suggest that a non-zero signal is expected. A negative result would mean that the Yukawa matrices and the $A$-terms are extremely well aligned indicating a special symmetry or a special nature of the SUSY breaking.

\footnotetext{
${ }^{6}$ We note that the constraints on $\left(\delta_{13}^{l}\right)_{L R}$ and $\left(\delta_{23}^{l}\right)_{L R}$ are trivially satisfied since their magnitude is bounded by $m_{\tau} / \tilde{m}$.
} 


\section{Prospects}

Different processes are sensitive to different chirality types of mass insertions, making it possible (at least in principle) to distinguish various supergravity textures. Below we give a few examples supporting this point.

The estimates (66) mean that SUSY flavour effects grow with quark masses. In particular, the magnitude of the LR mass insertions involving the third generation is enhanced in SUGRA models, which renders their effect potentially observable. They can significantly affect processes sensitive to LR mass insertions. Such processes are loop suppressed in the Standard Model and are usually due to penguin-type diagrams. Apart from $\epsilon^{\prime}$ and $\operatorname{Br}\left(\bar{B} \rightarrow X_{s} \gamma\right)$, a good example is the $B_{d}^{0} \rightarrow \phi K_{S}$ decay (for a recent discussion, see [51]). In this case, the decay is due to the $b \rightarrow s \bar{s} s$ transition and the ratio of the SUSY and SM decay amplitudes for squark and gluino masses of $500 \mathrm{GeV}$ is given by 52 ]

$$
\left(\frac{A^{\mathrm{SUSY}}}{A^{\mathrm{SM}}}\right)_{B \rightarrow \phi K_{S}} \simeq 100 \times\left[\left(\delta_{L R}^{d}\right)_{23}+\left(\delta_{R L}^{d}\right)_{23}\right]+0.2 \times\left[\left(\delta_{L L}^{d}\right)_{23}+\left(\delta_{R R}^{d}\right)_{23}\right] .
$$

Clearly, for $\left(\delta_{L R}^{d}\right)_{23} \sim 10^{-2}$ consistent with the $b \rightarrow s \gamma$ constraint, the SUSY and SM contributions are of similar magnitude. On the other hand, the chirality conserving insertions contribute far less significantly. Since in the SM the CP asymmetry in this decay coincides with that in the $B_{d}^{0} \rightarrow \psi K_{S}$ decay, it provides a sensitive probe for new physics contributions to the $b \rightarrow s \bar{s} s$ transition and, in particular, for supersymmetric contributions (see e.g. [53]). In the pseudoscalar channel of the $b \rightarrow s \bar{s} s$ transition, $B_{d}^{0} \rightarrow \eta^{\prime} K_{S}$, the SM predicts the same (within 5-10\%) CP asymmetry, whereas supersymmetry gives 52

$$
\left(\frac{A^{\mathrm{SUSY}}}{A^{\mathrm{SM}}}\right)_{B_{d}^{0} \rightarrow \eta^{\prime} K_{S}} \simeq 100 \times\left[\left(\delta_{L R}^{d}\right)_{23}-\left(\delta_{R L}^{d}\right)_{23}\right]+0.2 \times\left[\left(\delta_{L L}^{d}\right)_{23}-\left(\delta_{R R}^{d}\right)_{23}\right] .
$$

Thus, in SUGRA models one expects (correlated) deviations in both decays.

Another well known example of a process sensitive to LR mass insertions is the $b \rightarrow s \gamma$ transition (see e.g. [39]). As is clear from the bound (65), $\left(\delta_{23}^{d}\right)_{L R}$ of order $10^{-2}$, which is natural in SUGRA models, is sufficient to produce significant deviations from the SM prediction. On the other hand, to have a similar effect from the LL sector, a large $\left(\delta_{23}^{d}\right)_{L L}=\mathcal{O}(1)$ would be required. We note that the observation of a direct CP asymmetry in the $b \rightarrow s \gamma$ transition of order few percent would be a clean signal of new physics, since the well controlled SM prediction yields the asymmetry of less than one percent.

An example of a process particularly sensitive to LL and RR insertions is the $B_{s}^{0}-\bar{B}_{s}^{0}$ mixing. For moderate $\tan \beta$, the SUSY to SM ratio of the mixing amplitudes is [54]

$$
\begin{aligned}
\left(\frac{M_{12}^{\mathrm{SUSY}}}{M_{12}^{\mathrm{SM}}}\right)_{B_{s}^{0}-\bar{B}_{s}^{0}} & \simeq 1 \times\left[\left(\delta_{L L}^{d}\right)_{23}^{2}+\left(\delta_{R R}^{d}\right)_{23}^{2}\right]+30 \times\left[\left(\delta_{L R}^{d}\right)_{23}^{2}+\left(\delta_{R L}^{d}\right)_{23}^{2}\right] \\
& -45 \times\left[\left(\delta_{L R}^{d}\right)_{23}\left(\delta_{R L}^{d}\right)_{23}\right]-175 \times\left[\left(\delta_{L L}^{d}\right)_{23}\left(\delta_{R R}^{d}\right)_{23}\right]
\end{aligned}
$$


for gluino and squark masses of $500 \mathrm{GeV}$. At large $\tan \beta$, other contributions become important [55]. Given the limits on $\left(\delta_{L R}^{d}\right)_{23}$ from $b \rightarrow s \gamma$, the contribution of chirality changing mass insertions is negligible, while even a small chirality conserving insertions $\left(\delta_{L L}^{d}\right)_{23}$ and $\left(\delta_{R R}^{d}\right)_{23}$ of order $\mathcal{O}\left(10^{-1}\right)$ can induce a large departure of $\Delta M_{B_{s}}$ from the value predicted in the SM.

Essential for identifying the sources of flavour violation are correlations among different observables. To give an example, suppose that significant deviations from the SM predictions are found in the $B_{d}^{0} \rightarrow \phi K_{S}$ and $B_{d}^{0} \rightarrow \eta^{\prime} K_{S}$ decays. If they are due to LR/RL mass insertions, no deviation is expected in the $B_{s}^{0}-\bar{B}_{s}^{0}$ mixing. On the other hand, if the "anomaly" is due to LL/RR mass insertions, the $B_{s}^{0}-\bar{B}_{s}^{0}$ mixing should also be significantly affected. Thus, different SUGRA textures lead to different signatures. In principle, a more sophisticated network of correlations, including various other observables, can and should be developed. This, however, is beyond the scope of the present paper.

Significant SUSY effects can also be present in the top quark decays. The magnitude of $\left(\delta_{L R}^{u}\right)_{i 3}$ mass insertions is enhanced due to a large top Yukawa coupling. Since $\operatorname{BR}(t \rightarrow c \gamma)$ is particularly sensitive to LR insertions, $\operatorname{BR}(t \rightarrow c \gamma) \sim 10^{-6}\left(\delta_{L R}^{u}\right)_{23}^{2}$ [56], large departures from the $\mathrm{SM}$ prediction $\sim 10^{-13}$ are expected.

To conclude, we see that the current $B$-physics experiments are beginning to probe a natural range of supergravity non-universality in the A-terms. Some processes can further probe non-universality in the soft scalar masses, yet in this case it would be difficult to define a "natural" range due to larger model-dependence. Correlations among various observables can allow one to identify the source of flavour violation.

\section{Conclusions}

In this paper, we have presented a classification and analysis of flavour violating sources in general supergravity models. The current flavour physics data lead us to the following conclusions:

1. Flavour violation through the Kähler potential is disfavored, but room for flavour non-universality remains if the Kähler metric is diagonal. This often occurs in string models in which the Kähler potential is protected by string selection rules.

2. Departures from universality of order unity are allowed in the squark sector $A$-terms. Such departures are expected in typical string models.

3. A-terms in the charged slepton sector must be very well aligned with the lepton Yukawa matrix, which points at either a diagonal Yukawa matrix or universal $A$-terms.

4. A common and rather serious problem in supergravity models is the SUSY CP problem. 
5. The FCNC problem depends strongly on the Yukawa texture and is much milder for hierarchical Yukawa matrices. For diagonal squark masses, the problem essentially disappears if the Yukawa matrices are diagonalized by small angle rotations or if the CKM matrix derives entirely from rotations of the up type quarks. Thus, there exist varieties of textures ensuring sufficient suppression of FCNC.

6. Importance of SUSY flavour effects grows with quark masses.

7. Current $B$-physics experiments are beginning to probe a natural range of flavour non-universality in SUGRA models. Correlations among different observables can allow one to identify the source of flavour violation.

\begin{tabular}{|c||c|c|c|}
\hline Texture & Hierarchical Yukawas & Democratic Yukawas & Mode \\
\hline (A) & no problem & no problem & $?$ \\
(B) & no problem & problem & $b \rightarrow s \gamma, b \rightarrow s \bar{s} s$ \\
(C) & no serious problem & problem & $B_{s}^{0}-\bar{B}_{s}^{0}$ \\
(D) & no serious problem & problem & see (B), (C) \\
(E) & problem & problem & see (B), (C) \\
\hline
\end{tabular}

Table 3: Compatibility of the supergravity soft term textures with the Yukawa textures. Possible detection modes are also indicated.

It is important to note the difference between the flavour structure of the Standard Model and that of supergravity soft terms. In the former, we encounter a hierarchical pattern of the Yukawa couplings. In supergravity, the soft terms are logarithmic derivatives of hierarchical quantities. As a result, $m_{i}^{2}$ and $\tilde{A}_{i j}$ are expected to be of the same order, in sharp contrast with the Yukawa couplings.

Finally, from the point of view of avoiding the FCNC problem, Ansätze (A) and (B) are clearly preferred. Ansatz (B) represents a rather typical prediction of string models (e.g. heterotic string, intersecting branes, etc.). Textures (C) and (D) are also allowed under the condition of some degeneracy in the (12) block. Texture (E) is clearly disfavored. These conclusions are summarized in Table 3. The main message is that the supergravity textures are not necessarily restricted to the universal one and can be quite rich, depending on the mechanism generating the Yukawa matrices. These flavour structures can be probed experimentally.

\section{Acknowledgements}

This work has been supported in part by the RTN European Program MRTN-CT-2004503369. P.H.Ch. and S.P. were supported by the Polish State Committee for Scientific Research Grants 2 P03B 04024 for 2003-2005 and 2 P03B 12924 for 2003-2005, respectively. P.H.Ch. would like to thank the CERN Theory Group for hospitality during the completion of this work. This work was started during S.P.'s visit to the University of Hamburg. The visit was possible owing to "Forschung-Preis" of the A. von Humboldt 
Foundation. S.P. thanks Jan Louis for his hospitality. This collaboration was stimulated by an ENTApP sponsored visitor's programme on dark matter at CERN, 17 January - 4 February 2005.

\section{References}

[1] H. P. Nilles, Phys. Rept. 110 (1984) 1; S. Weinberg, "The quantum theory of fields. Vol. 3: Supersymmetry," Cambridge University Press, Cambridge, 2000.

[2] S. K. Soni and H. A. Weldon, Phys. Lett. B 126, 215 (1983).

[3] V. S. Kaplunovsky and J. Louis, Phys. Lett. B 306, 269 (1993).

[4] A. Brignole, L. E. Ibanez and C. Munoz, "Soft supersymmetry-breaking terms from supergravity and superstring models," hep-ph/9707209

[5] D. J. H. Chung, L. L. Everett, G. L. Kane, S. F. King, J. Lykken and L. T. Wang, "The soft supersymmetry-breaking Lagrangian: Theory and applications," hep-ph/0312378.

[6] O. Lebedev, Phys. Rev. D 67, 015013 (2003).

[7] P. Nath, R. Arnowitt and A. H. Chamseddine, "Applied N=1 Supergravity," NUB2613, Lectures given at Summer Workshop on Particle Physics, Trieste, Italy, Jun 20 - Jul 29, 1983.

[8] S. Abel, S. Khalil and O. Lebedev, Phys. Rev. Lett. 89, 121601 (2002).

[9] S. Hamidi and C. Vafa, Nucl. Phys. B 279, 465 (1987); L. J. Dixon, D. Friedan, E. J. Martinec and S. H. Shenker, Nucl. Phys. B 282, 13 (1987).

[10] L. E. Ibanez, Phys. Lett. B 181, 269 (1986).

[11] J. A. Casas and C. Munoz, Nucl. Phys. B 332, 189 (1990) [Erratum-ibid. B 340, 280 (1990)].

[12] P. Brax, U. Ellwanger and C. A. Savoy, Phys. Lett. B 347, 269 (1995).

[13] O. Lebedev, Phys. Lett. B 521, 71 (2001).

[14] S. Khalil, O. Lebedev and S. Morris, Phys. Rev. D 65, 115014 (2002).

[15] L. E. Ibanez and D. Lust, Nucl. Phys. B 382, 305 (1992).

[16] R. Blumenhagen, M. Cvetic, P. Langacker and G. Shiu, "Toward Realistic Intersecting D-Brane Models," hep-th/0502005.

[17] R. Blumenhagen, L. Goerlich, B. Kors and D. Lust, JHEP 0010, 006 (2000). 
[18] G. Aldazabal, S. Franco, L. E. Ibanez, R. Rabadan and A. M. Uranga, J. Math. Phys. 42, 3103 (2001).

[19] D. Cremades, L. E. Ibanez and F. Marchesano, JHEP 0307, 038 (2003). For realistic examples, see S. A. Abel, O. Lebedev and J. Santiago, Nucl. Phys. B 696, 141 (2004).

[20] D. Lust, P. Mayr, R. Richter and S. Stieberger, Nucl. Phys. B 696, 205 (2004).

[21] C. D. Froggatt and H. B. Nielsen, Nucl. Phys. B 147, 277 (1979).

[22] S. Khalil, T. Kobayashi and A. Masiero, Phys. Rev. D 60, 075003 (1999).

[23] K. Choi, J. S. Lee and C. Munoz, Phys. Rev. Lett. 80, 3686 (1998).

[24] A. Brignole, L. E. Ibanez and C. Munoz, Nucl. Phys. B 422, 125 (1994) [Erratumibid. B 436, 747 (1995)]; D. Choudhury, F. Eberlein, A. Konig, J. Louis and S. Pokorski, Phys. Lett. B 342, 180 (1995); P. Brax and C. A. Savoy, Nucl. Phys. B 447, 227 (1995).

[25] L. J. Hall, V. A. Kostelecky and S. Raby, Nucl. Phys. B 267, 415 (1986).

[26] M. Misiak, S. Pokorski and J. Rosiek, Adv. Ser. Direct. High Energy Phys. 15, 795 (1998).

[27] E. Dudas, S. Pokorski and C. A. Savoy, Phys. Lett. B 369, 255 (1996); E. Dudas, C. Grojean, S. Pokorski and C. A. Savoy, Nucl. Phys. B 481, 85 (1996).

[28] S. A. Abel and G. Servant, Nucl. Phys. B 597, 3 (2001).

[29] G. G. Ross and O. Vives, Phys. Rev. D 67, 095013 (2003).

[30] T. Kobayashi and O. Vives, Phys. Lett. B 506, 323 (2001).

[31] J. A. Casas and S. Dimopoulos, Phys. Lett. B 387, 107 (1996).

[32] For a recent study, see P. Ko, T. Kobayashi and J. h. Park, Phys. Lett. B 598, 263 (2004).

[33] A. E. Faraggi and E. Halyo, Nucl. Phys. B 416, 63 (1994); T. Kobayashi, Phys. Lett. B 358, 253 (1995).

[34] P. H. Chankowski, K. Kowalska, S. Lavignac and S. Pokorski, "Update on fermion mass models with an anomalous horizontal U(1) symmetry," hep-ph/0501071.

[35] D. Chang, A. Masiero and H. Murayama, Phys. Rev. D 67, 075013 (2003).

[36] G. C. Branco, J. I. Silva-Marcos and M. N. Rebelo, Phys. Lett. B 237, 446 (1990).

[37] H. Fritzsch and J. Plankl, Phys. Lett. B 237, 451 (1990).

[38] S. Abel, G. C. Branco and S. Khalil, Phys. Lett. B 569, 14 (2003). 
[39] F. Gabbiani, E. Gabrielli, A. Masiero and L. Silvestrini, Nucl. Phys. B 477, 321 (1996); J. S. Hagelin, S. Kelley and T. Tanaka, Nucl. Phys. B 415, 293 (1994).

[40] T. Falk, K. A. Olive, M. Pospelov and R. Roiban, Nucl. Phys. B 560, 3 (1999).

[41] S. Abel, S. Khalil and O. Lebedev, Nucl. Phys. B 606, 151 (2001).

[42] J. R. Ellis, S. Ferrara and D. V. Nanopoulos, Phys. Lett. B 114, 231 (1982); W. Buchmuller and D. Wyler, Phys. Lett. B 121, 321 (1983); J. Polchinski and M. B. Wise, Phys. Lett. B 125, 393 (1983).

[43] O. Lebedev and S. Morris, JHEP 0208, 007 (2002).

[44] L. E. Ibanez, "The fluxed MSSM," hep-ph/0408064.

[45] S. Khalil and O. Lebedev, Phys. Lett. B 515, 387 (2001).

[46] A. Masiero and H. Murayama, Phys. Rev. Lett. 83, 907 (1999).

[47] G. Colangelo and G. Isidori, JHEP 9809, 009 (1998).

[48] D. Chang, W. F. Chang, W. Y. Keung, N. Sinha and R. Sinha, Phys. Rev. D 65, 055010 (2002).

[49] E. Gabrielli and S. Khalil, Phys. Rev. D 67, 015008 (2003).

[50] For an update and prospects, see e.g. S. Lavignac, "Flavour and CP violation in the lepton sector and new physics," eConf C030603, VEN04 (2003), hep-ph/0312309.

[51] G. L. Kane, P. Ko, H. b. Wang, C. Kolda, J. h. Park and L. T. Wang, Phys. Rev. Lett. 90, 141803 (2003).

[52] S. Khalil and E. Kou, Phys. Rev. Lett. 91, 241602 (2003).

[53] Y. Nir, "CP violation: The CKM matrix and new physics," Nucl. Phys. Proc. Suppl. 117, 111 (2003); Y. Grossman, "Footprints of new physics in the B system," AIP Conf. Proc. 722 (2004) 255.

[54] P. Ball, S. Khalil and E. Kou, Phys. Rev. D 69, 115011 (2004).

[55] A. J. Buras, P. H. Chankowski, J. Rosiek and L. Sławianowska, Phys. Lett. B 546 (2002) 96; Nucl. Phys. B 659 (2003) 3; P. H. Chankowski and J. Rosiek, Acta Phys. Polon. B 33 (2002) 2329; A. Dedes, Mod. Phys. Lett. A 18 (2003) 2627.

[56] D. Delepine and S. Khalil, Phys. Lett. B 599, 62 (2004). 

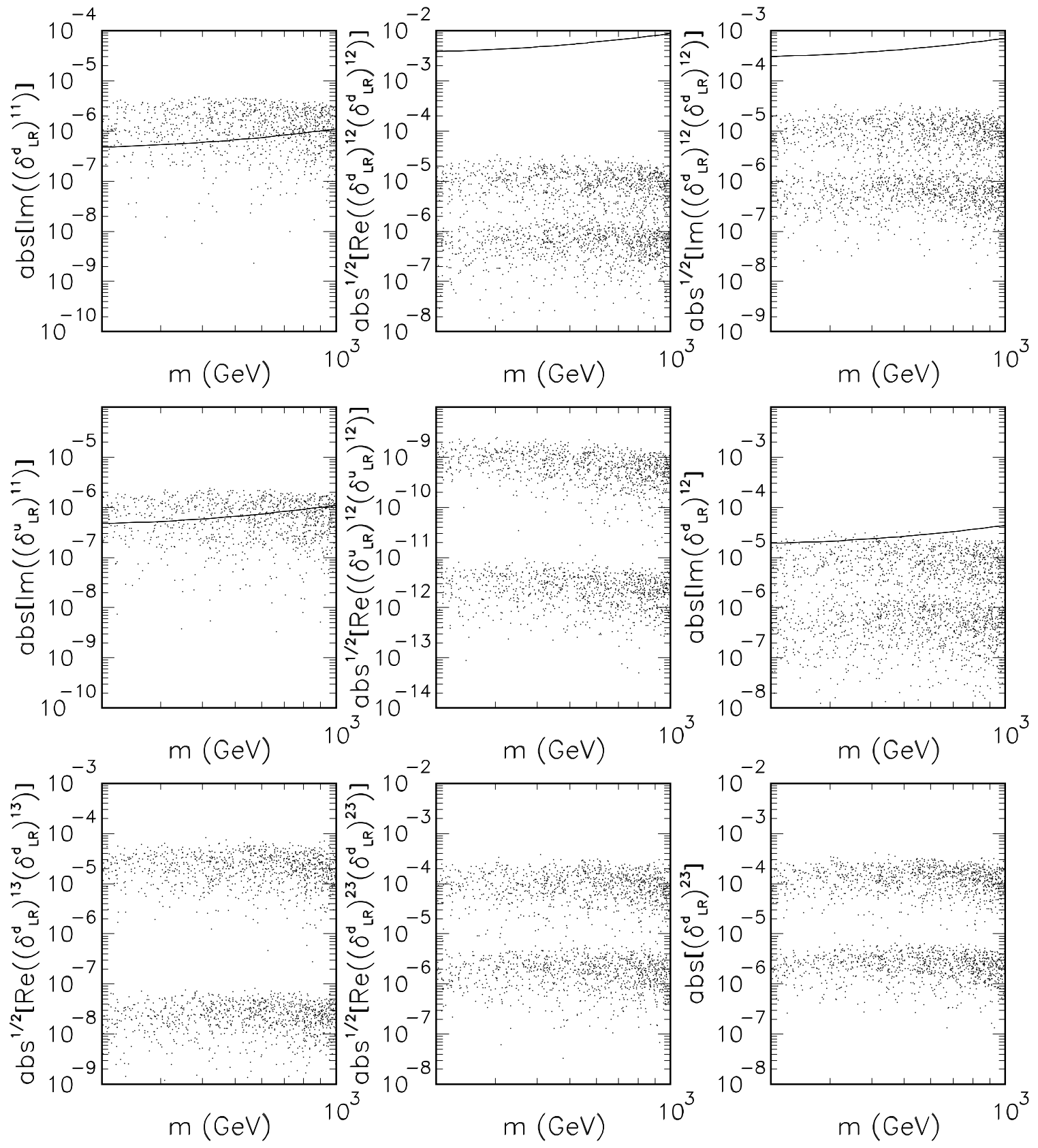

Figure 1: Phenomenologically relevant combinations of the insertions $\left(\delta_{L R}^{d, u}\right)^{J I}$ and $\left(\delta_{R L}^{d, u}\right)^{J I}$ for the hierarchical Yukawa texture (i) as a function of the universal mass scale $m=2 \mathrm{~A}$ with $M_{1 / 2}=200 \mathrm{GeV}, \tan \beta=15$ and order one $\tilde{A}_{i j}^{u, d}$ generated randomly. Each panel shows both the LR and RL mass insertions. The experimental limit is represented by the curve. 

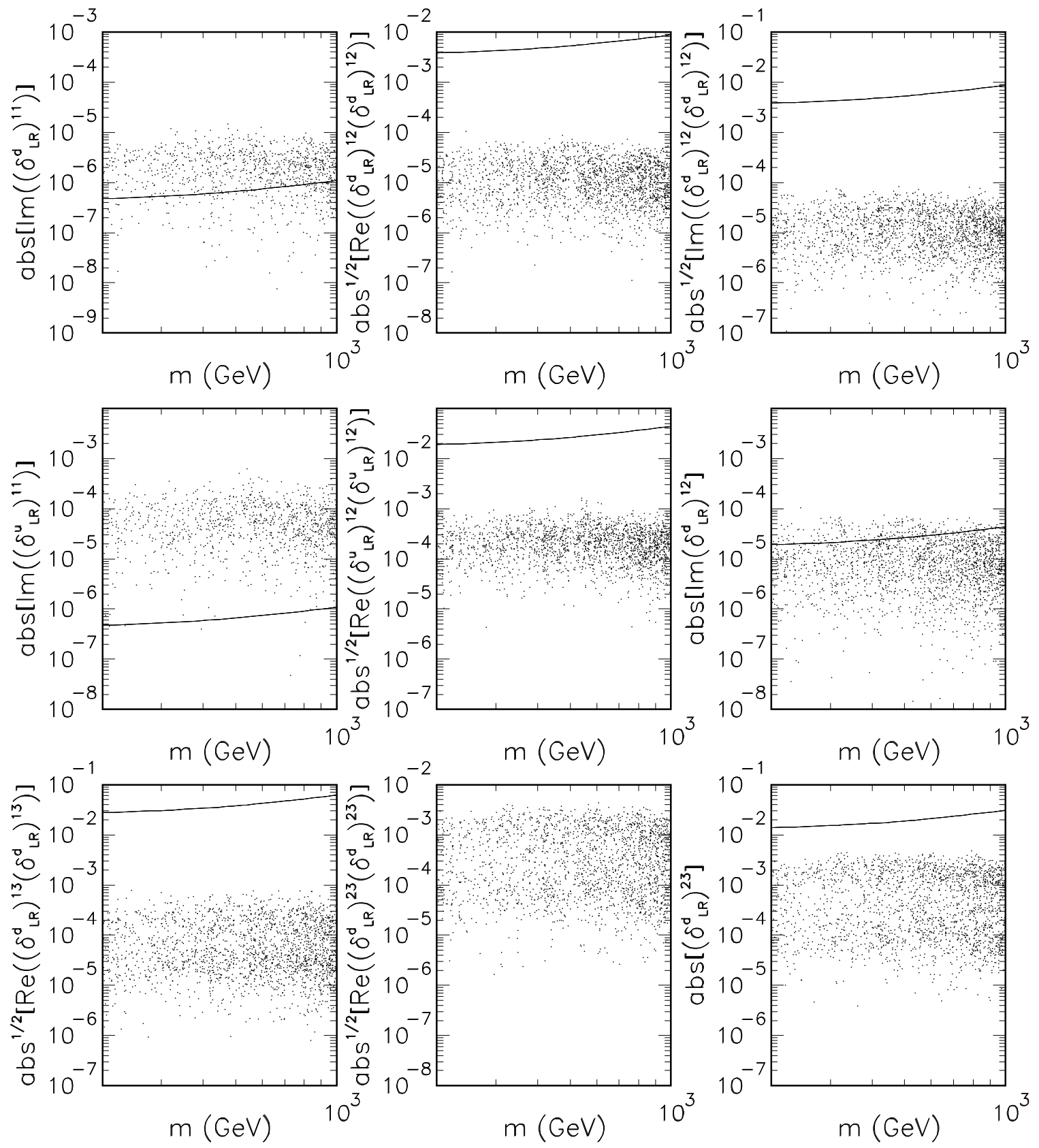

Figure 2: As in Figure 1 but for the hierarchical Yukawa texture (ii). 

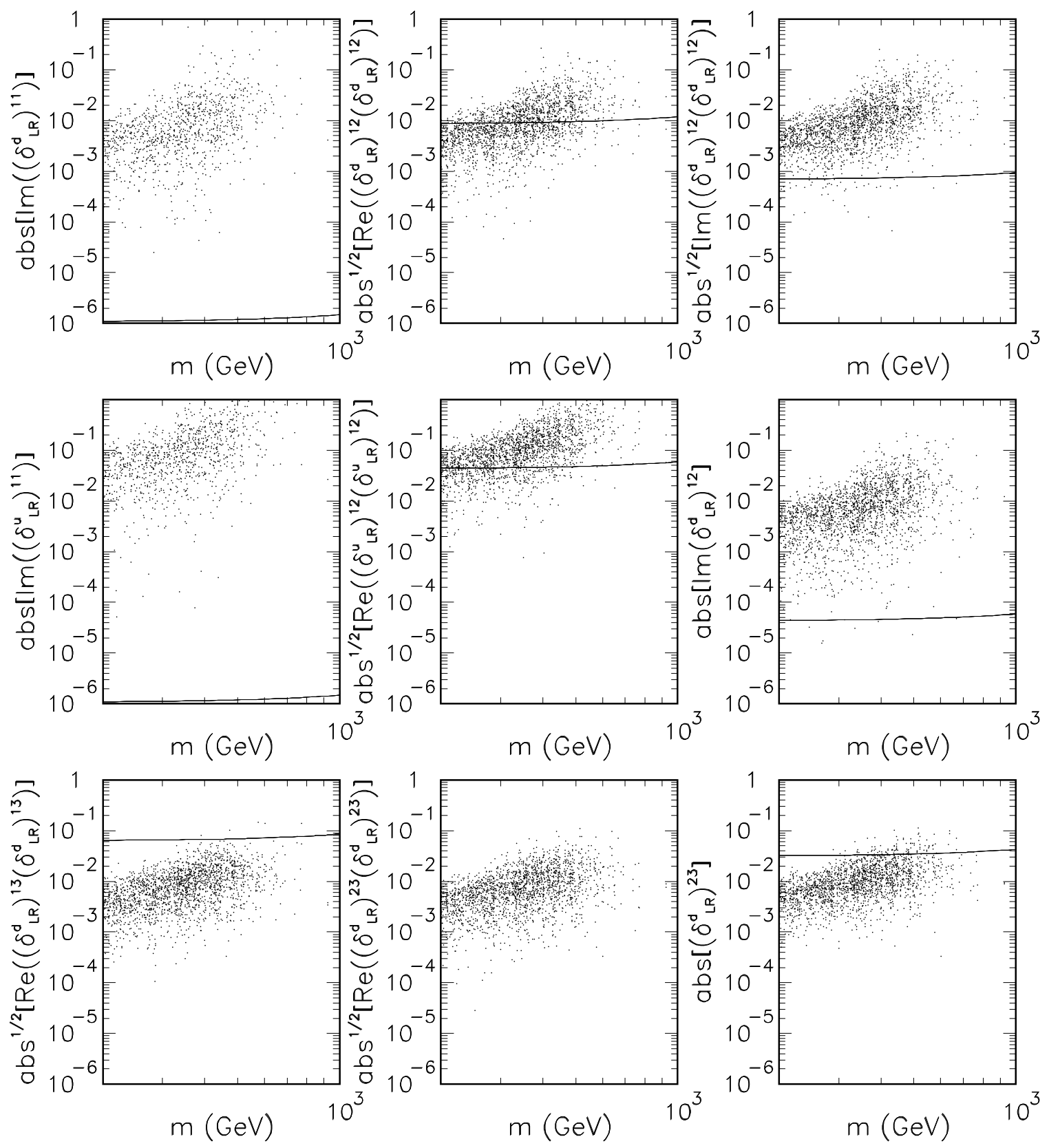

Figure 3: As in Figure 1 but for the democratic Yukawa texture (iii) and $M_{1 / 2}=500 \mathrm{GeV}$. 

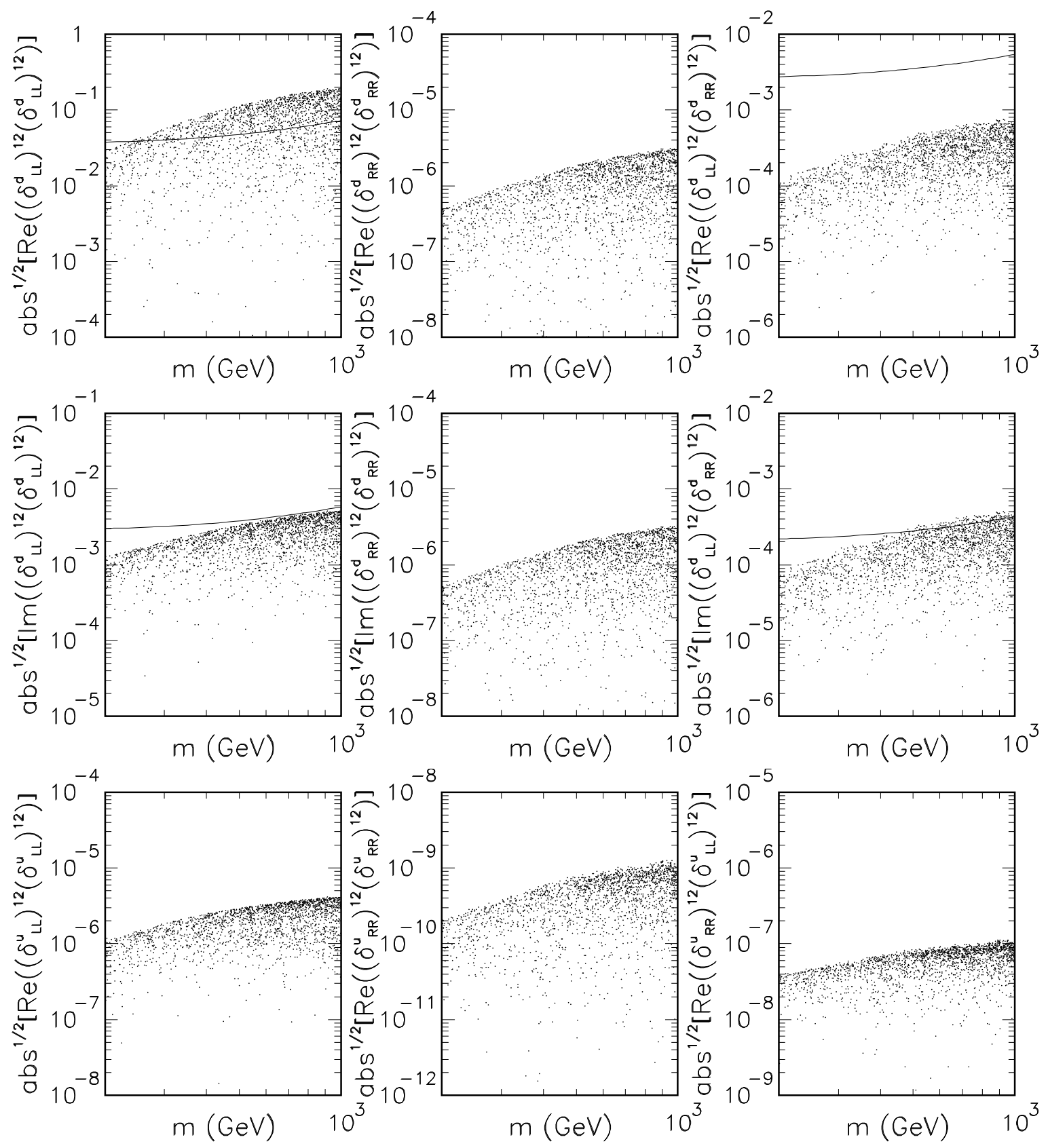

Figure 4: Phenomenologically relevant combinations of the insertions $\left(\delta_{L L}^{d, u}\right)^{12}$ and $\left(\delta_{R R}^{d, u}\right)^{12}$ for the hierarchical Yukawa texture (i) as a function of the universal mass scale $m \equiv$ $m_{2}=m_{3}$ with $M_{1 / 2}=200 \mathrm{GeV}, A=0, \tan \beta=15$ and $m_{1}$ varied randomly in the range $m / 2 \div m$. The experimental limit is represented by the curve. 

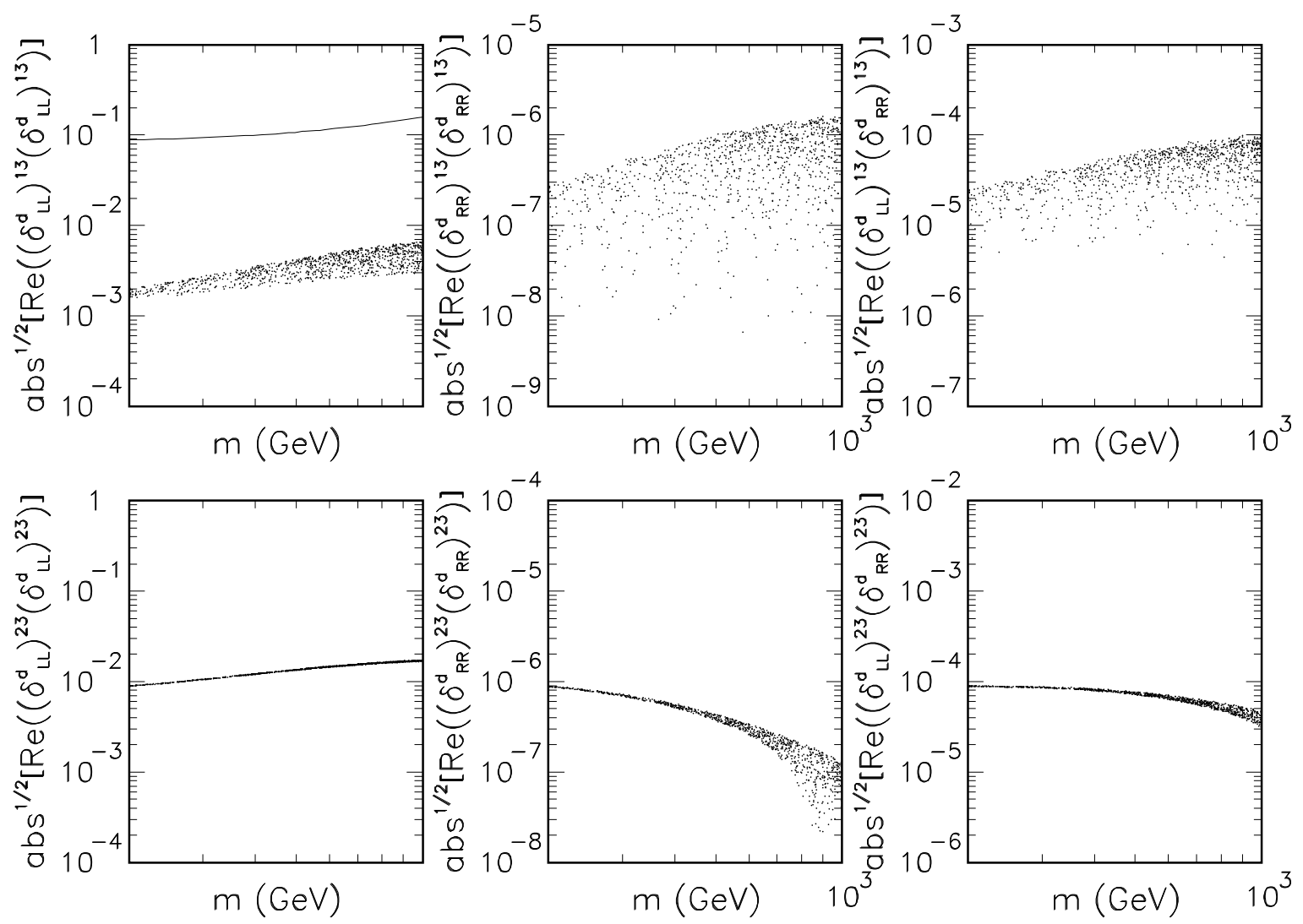

Figure 5: Phenomenologically relevant combinations of the insertions $\left(\delta_{L L}^{d}\right)^{13},\left(\delta_{R R}^{d}\right)^{13}$, $\left(\delta_{L L}^{d}\right)^{23},\left(\delta_{R R}^{d}\right)^{23}$ for the hierarchical Yukawa texture (i) as a function of the universal mass scale $m \equiv m_{2}=m_{3}$ with $M_{1 / 2}=200 \mathrm{GeV}, A=0, \tan \beta=15$ and $m_{1}$ varied randomly in the range $m / 2 \div m$. The experimental limit is represented by the curve. 

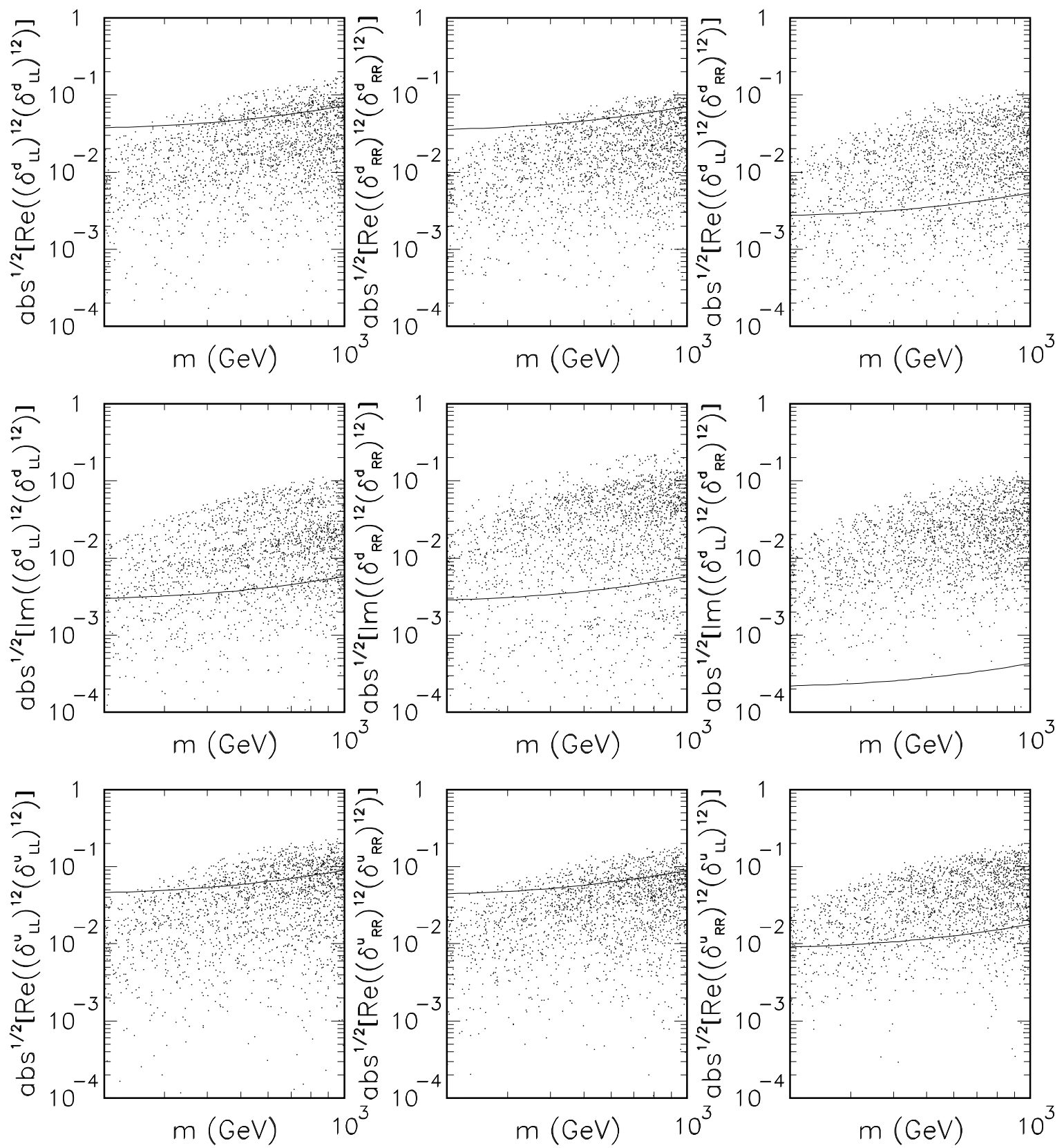

Figure 6: As in Figure \but for the hierarchical Yukawa texture (ii). 

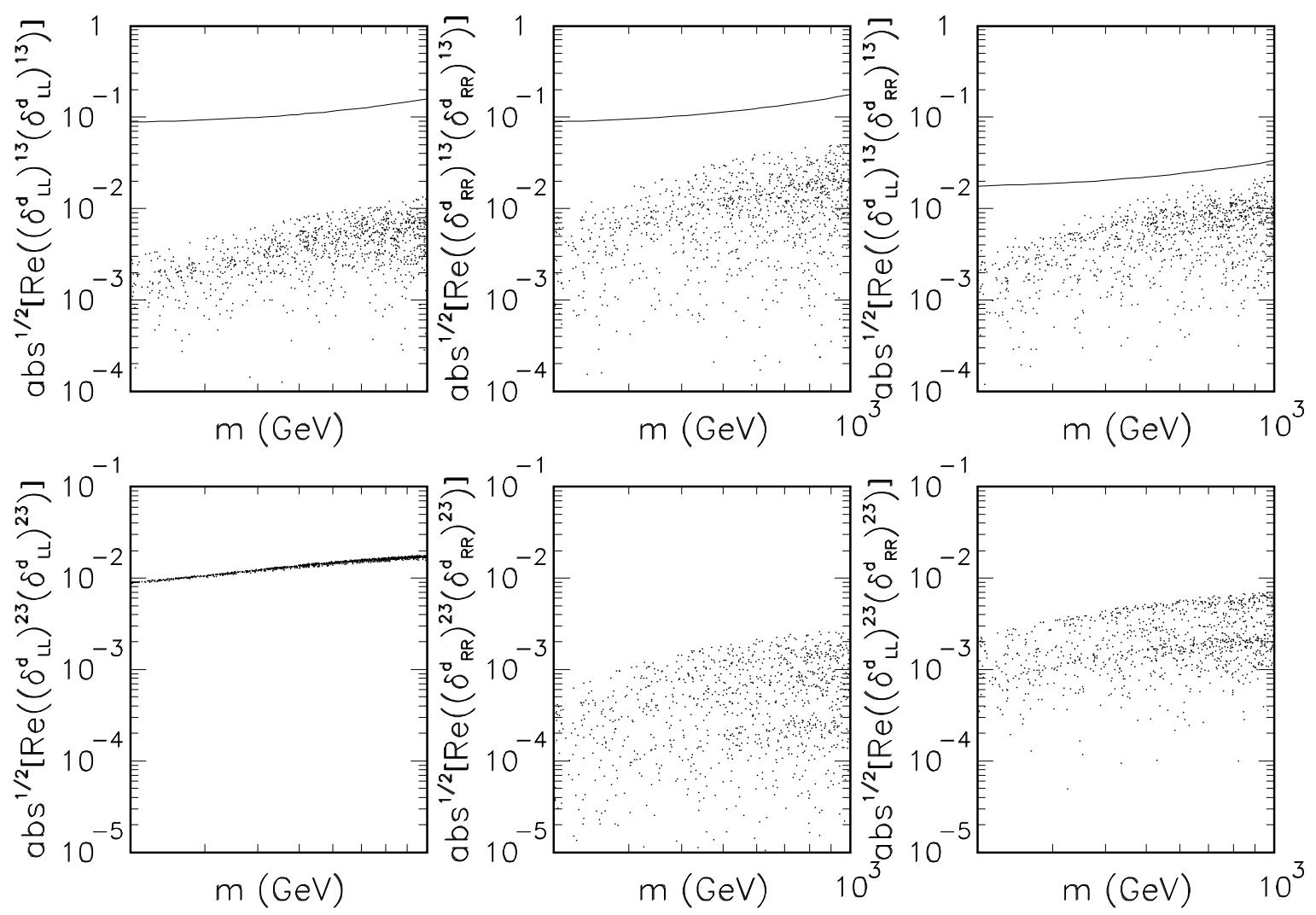

Figure 7: As in Figure 5 but for the hierarchical Yukawa texture (ii). 

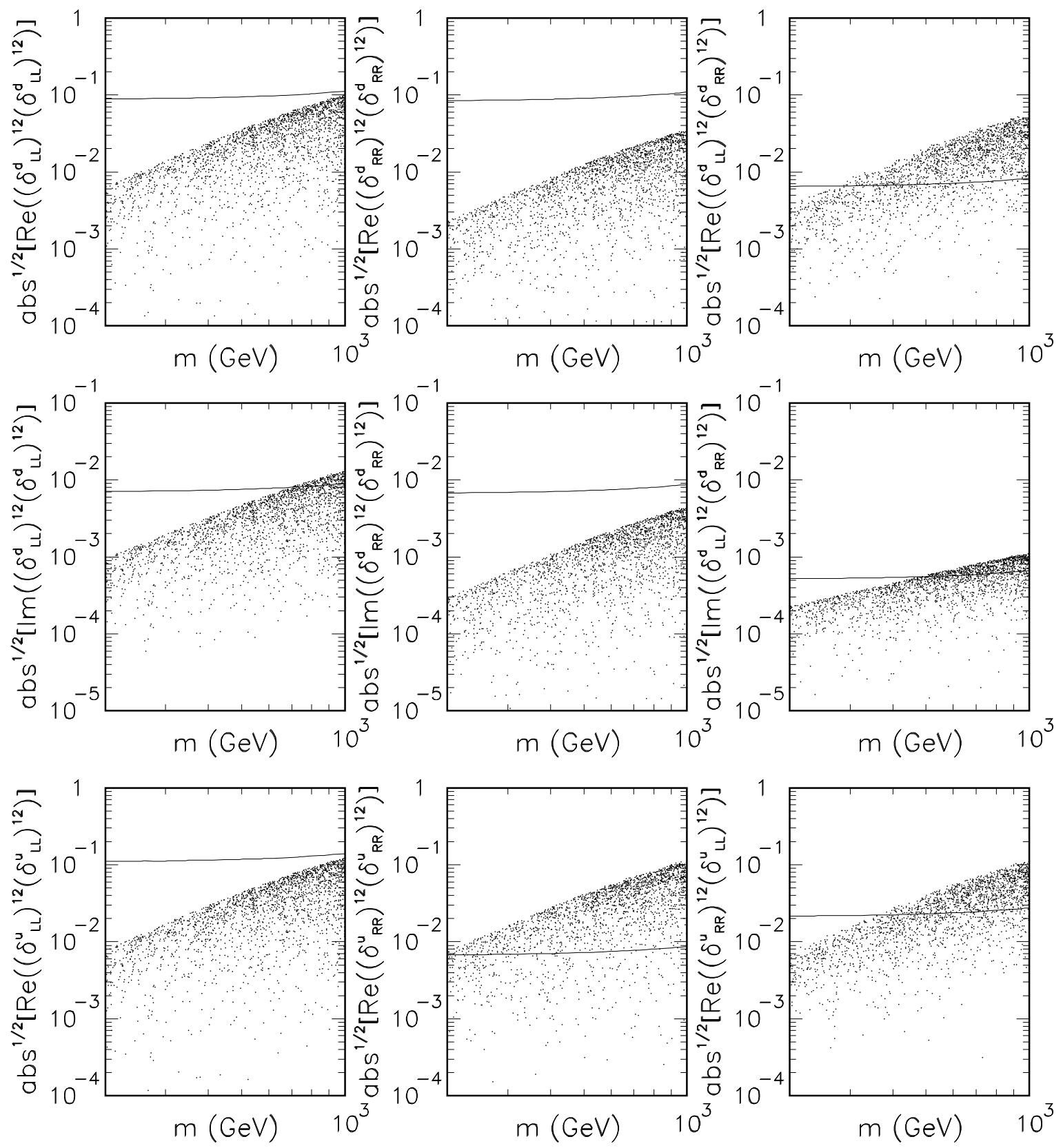

Figure 8: As in Figure团 but for the democratic Yukawa texture (iii) and $M_{1 / 2}=500 \mathrm{GeV}$. 

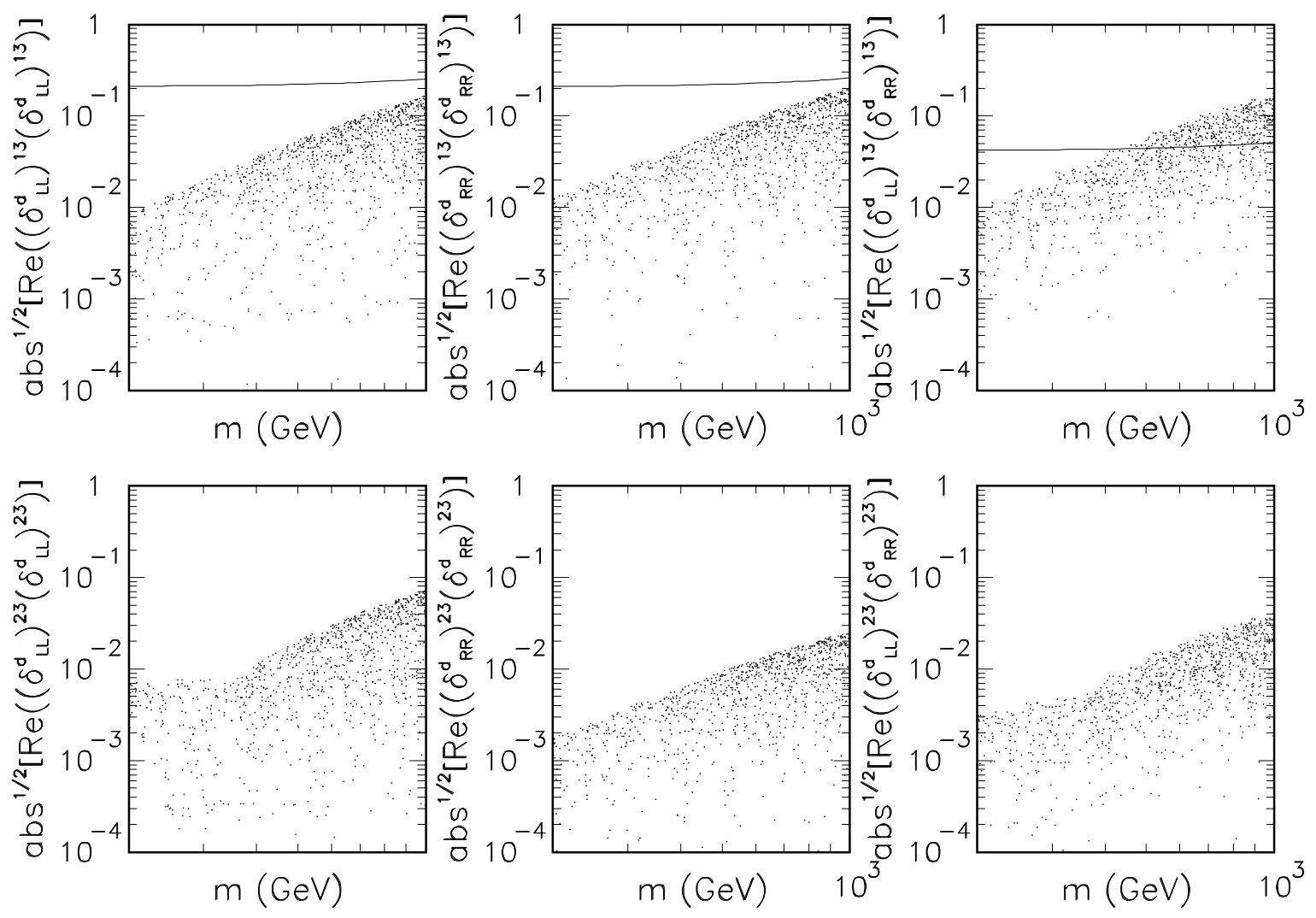

Figure 9: As in Figure 5 but for the democratic Yukawa texture (iii) and $M_{1 / 2}=500 \mathrm{GeV}$. 

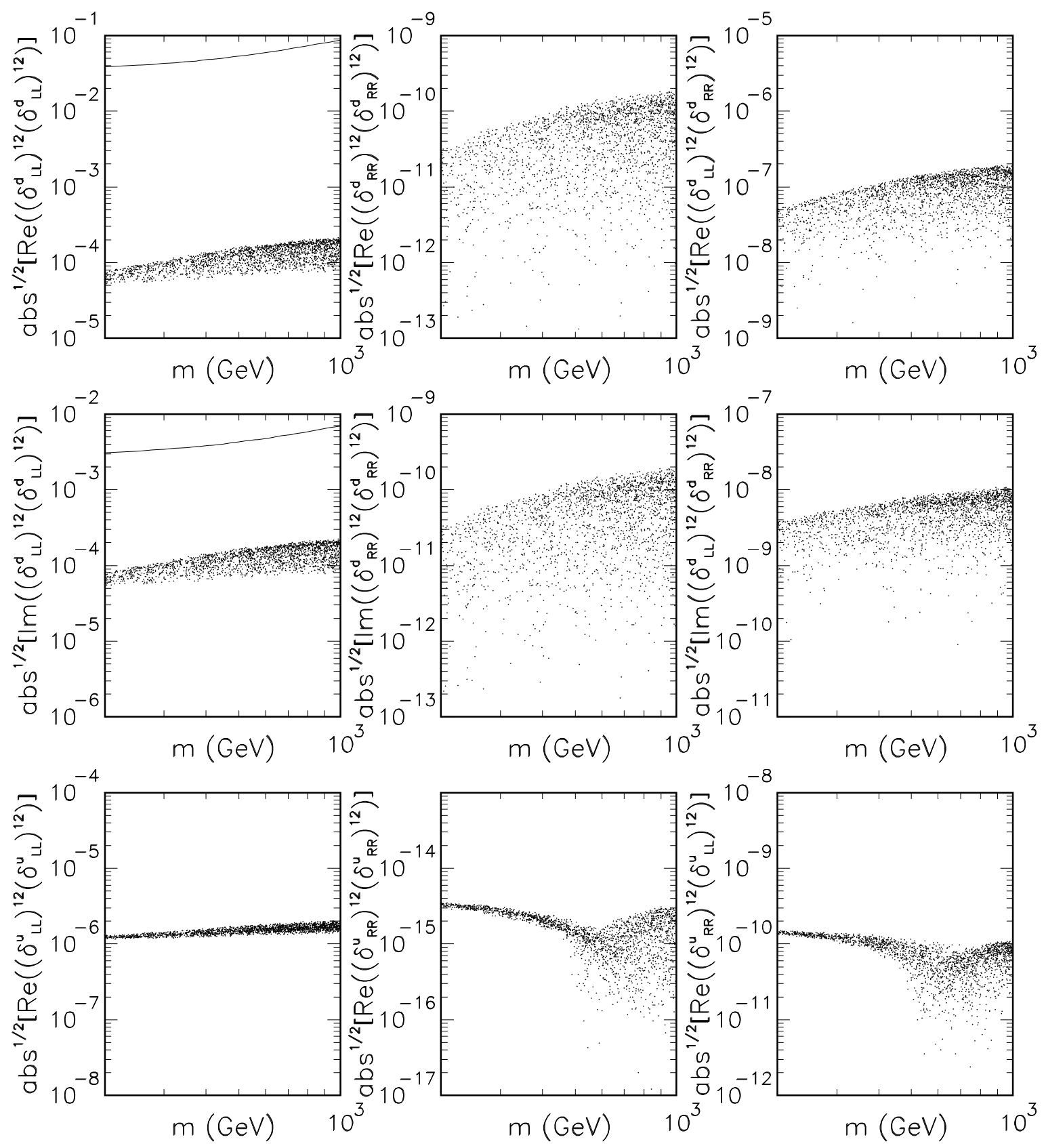

Figure 10: Phenomenologically relevant combinations of the insertions $\left(\delta_{L L}^{d, u}\right)^{12}$ and $\left(\delta_{R R}^{d, u}\right)^{12}$ for the hierarchical Yukawa texture (i) as a function of the universal mass scale $m \equiv$ $m_{1}=m_{2}$ with $M_{1 / 2}=200 \mathrm{GeV}, A=0, \tan \beta=15$ and $m_{3}$ varied randomly in the range $m / 2 \div m$. The experimental limit is represented by the curve. 

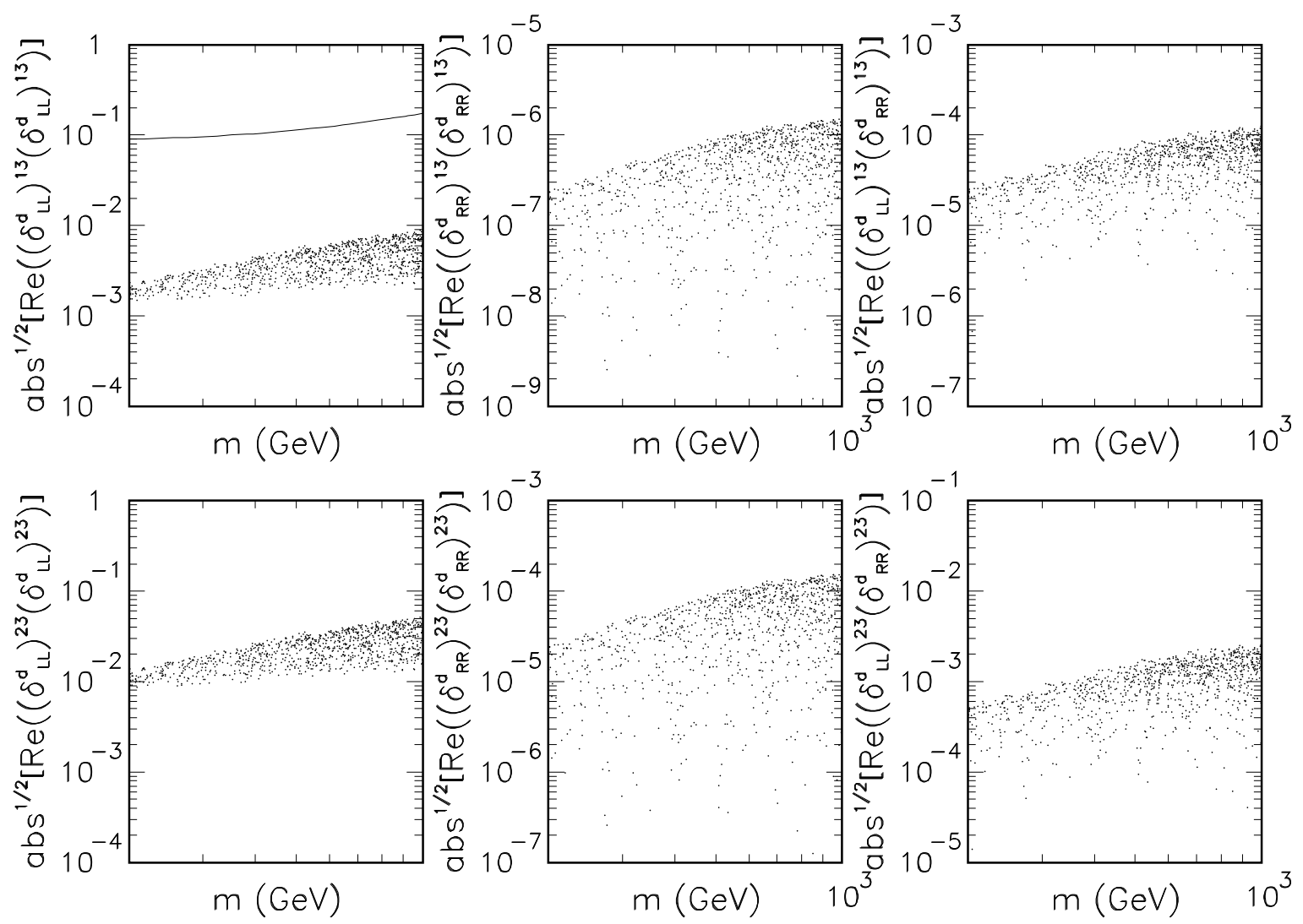

Figure 11: Phenomenologically relevant combinations of the insertions $\left(\delta_{L L}^{d}\right)^{13},\left(\delta_{R R}^{d}\right)^{13}$, $\left(\delta_{L L}^{d}\right)^{23},\left(\delta_{R R}^{d}\right)^{23}$ for the hierarchical Yukawa texture (i) as a function of the universal mass scale $m \equiv m_{1}=m_{2}$ with $M_{1 / 2}=200 \mathrm{GeV}, A=0, \tan \beta=15$ and $m_{3}$ varied randomly in the range $m / 2 \div m$. The experimental limit is represented by the curve. 

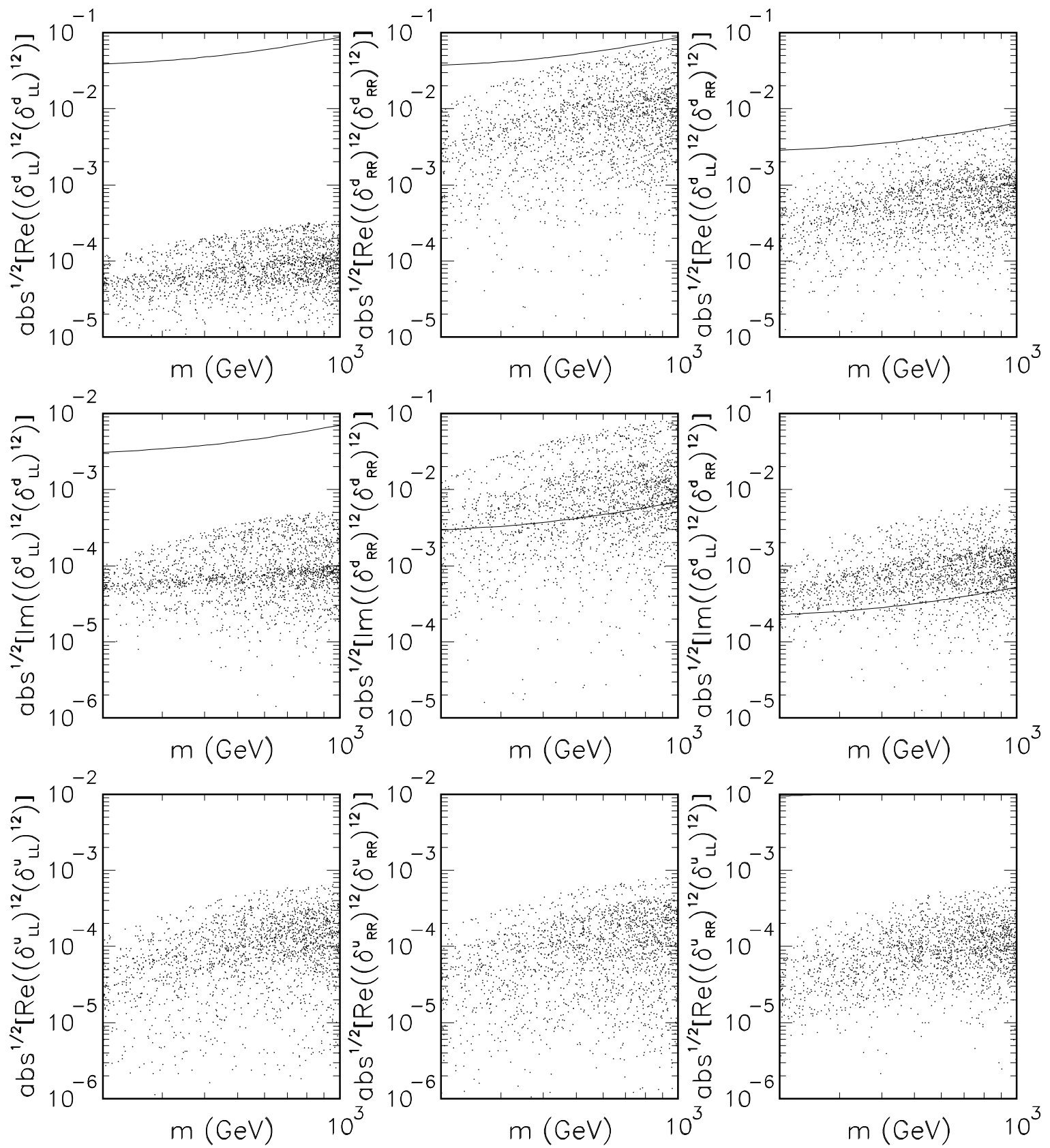

Figure 12: As in Figure 10 but for the hierarchical Yukawa texture (ii). 

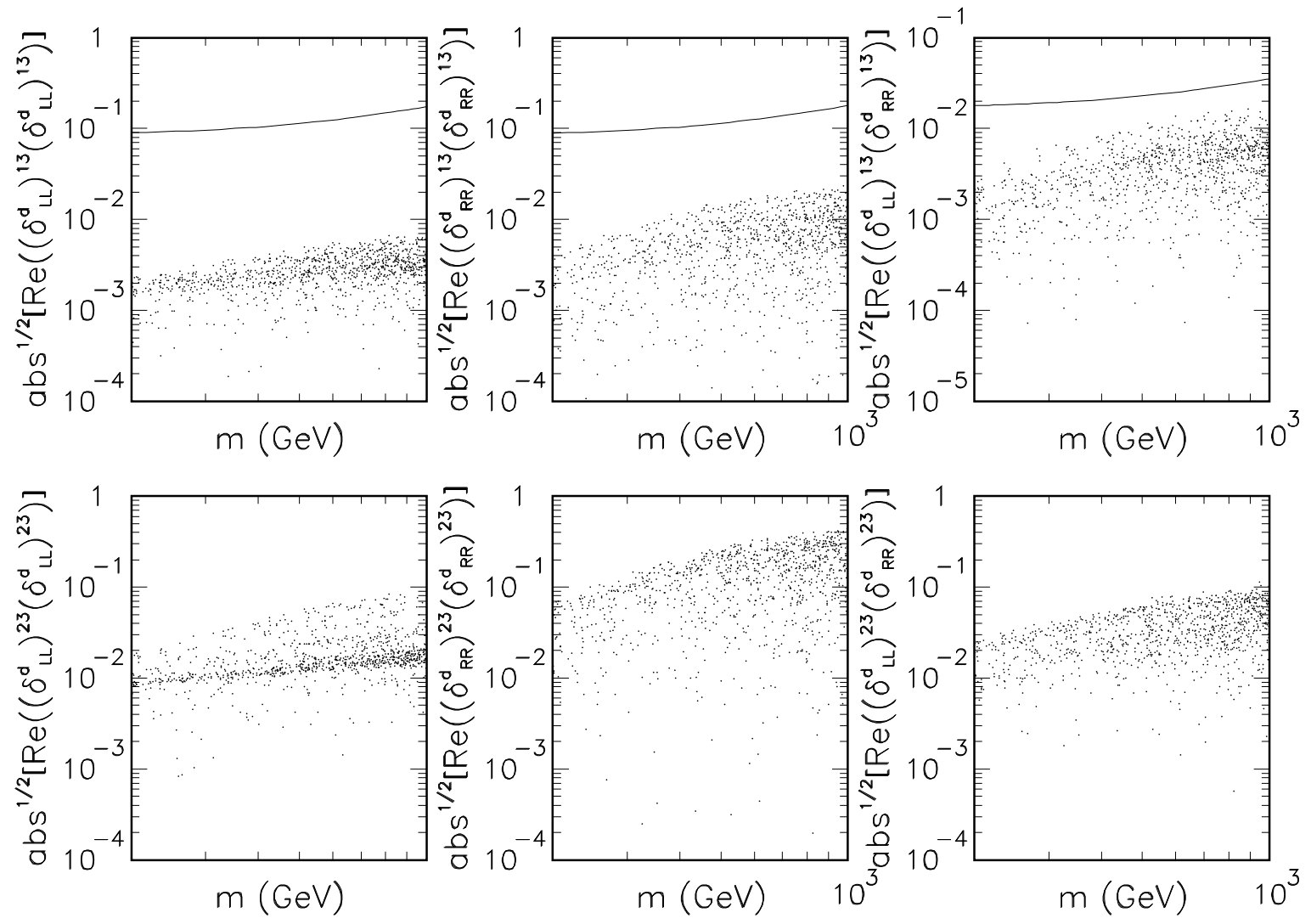

Figure 13: As in Figure 11 but for the hierarchical Yukawa texture (ii). 

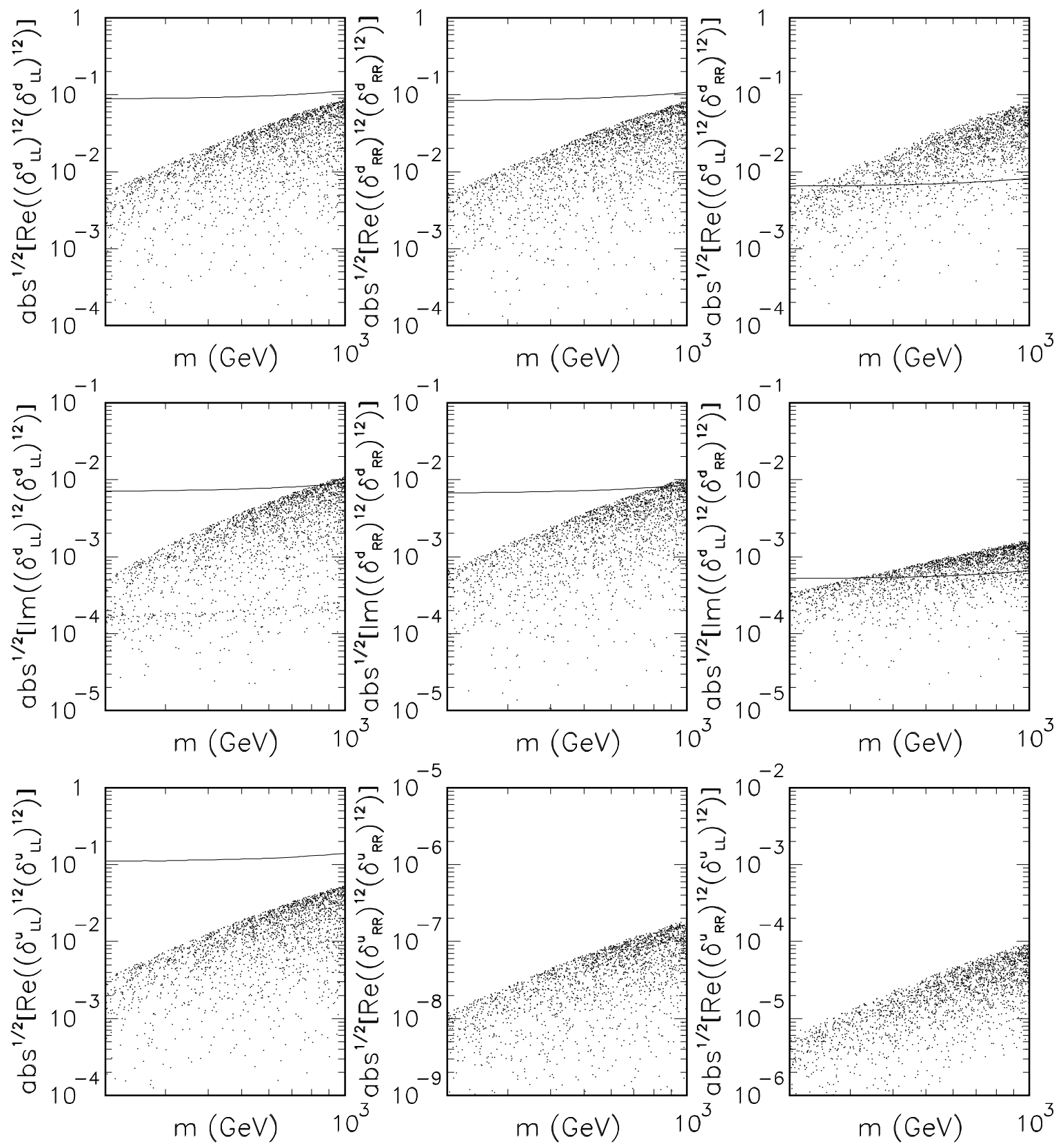

Figure 14: As in Figure 10 but for the democratic Yukawa texture (iii) and $M_{1 / 2}=$ $500 \mathrm{GeV}$. 

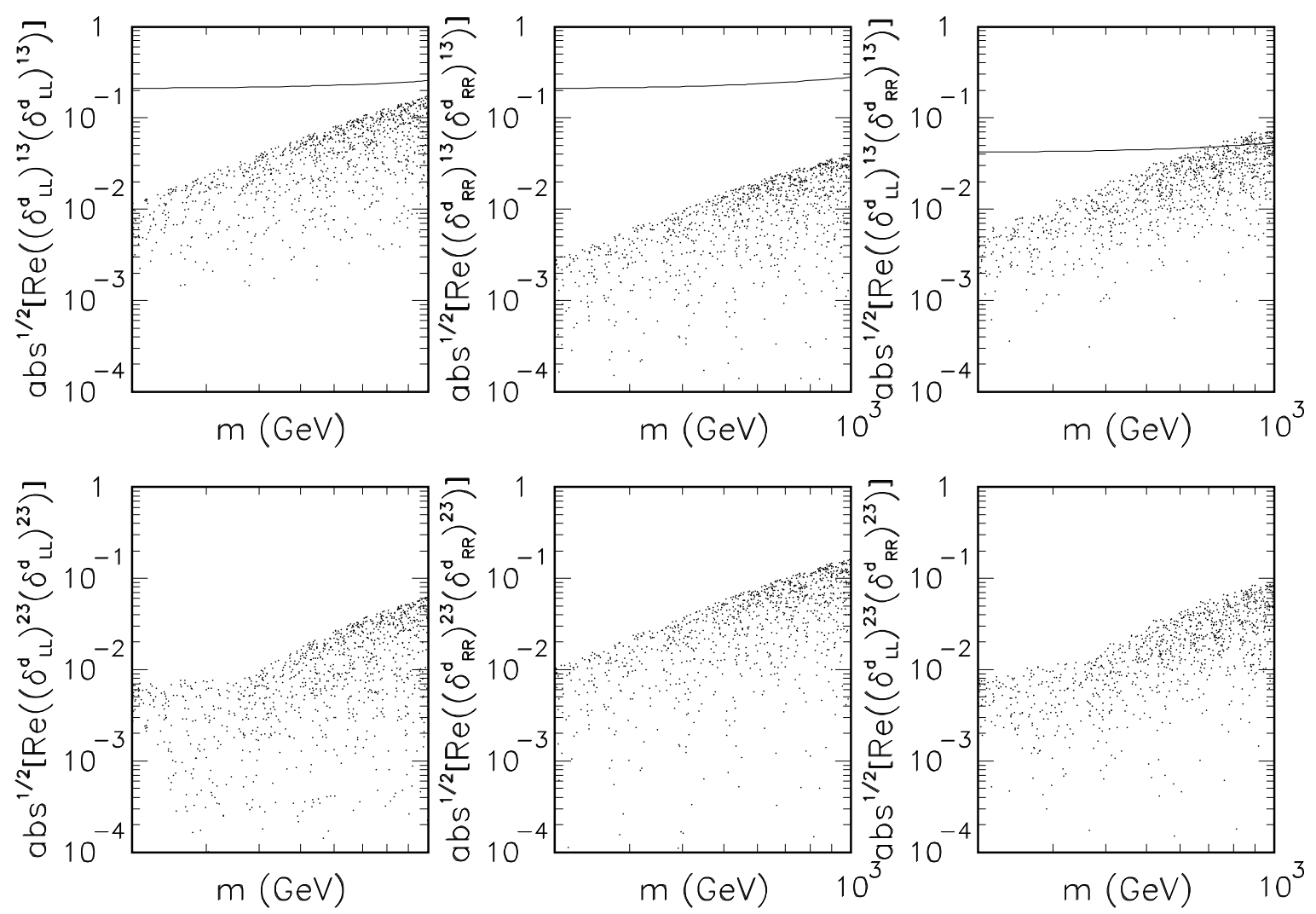

Figure 15: As in Figure 11 but for the democratic Yukawa texture (iii) and $M_{1 / 2}=$ $500 \mathrm{GeV}$. 